\title{
Superconformal structures on the three-sphere
}

\author{
Sergei M. Kuzenko ${ }^{a}$ and D. Sorokin ${ }^{b}$ \\ ${ }^{a}$ School of Physics M013, The University of Western Australia, \\ 35 Stirling Highway, Crawley W.A. 6009, Australia \\ ${ }^{b}$ Sezione di Padova, Istituto Nazionale di Fisica Nucleare (INFN), \\ via F. Marzolo 8, 35131 Padova, Italy \\ E-mail: sergei.kuzenko@uwa.edu.au, Dmitri.Sorokin@pd.infn.it
}

ABSTRACT: With the motivation to develop superconformal field theory on $S^{3}$, we introduce a $2 n$-extended supersphere $S^{3 \mid 4 n}$, with $n=1,2, \ldots$, as a homogeneous space of the three-dimensional Euclidean superconformal group $\operatorname{OSp}(2 n \mid 2,2)$ such that its bosonic body is $S^{3}$. Supertwistor and bi-supertwistor realizations of $S^{3 \mid 4 n}$ are derived. We study in detail the $n=1$ case, which is unique in the sense that the $R$-symmetry subgroup $\mathrm{SO}^{*}(2 n)$ of the superconformal group is compact only for $n=1$. In particular, we show that the $\operatorname{OSp}(2 \mid 2,2)$ transformations preserve the chiral subspace of $S^{3 \mid 4}$. Several supercoset realizations of $S^{3 \mid 4 n}$ are presented. Harmonic/projective extensions of the supersphere by auxiliary bosonic fibre directions are sketched.

KEYwords: Extended Supersymmetry, Superspaces

ArXiv EPRINT: 1406.7090 


\section{Contents}

1 Introduction 2

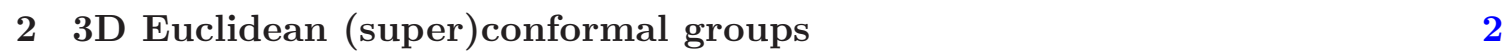

2.1 The conformal group 3

2.2 The superconformal group 4

2.3 The superconformal algebra 5

3 The three-sphere as a conformal space $\quad 6$

$\begin{array}{ll}3.1 \text { Twistor realization of the three-sphere } & 6\end{array}$

3.2 Real structure 8

3.3 Bitwistor realization 8

$\begin{array}{ll}3.4 & \text { Atlas on the three-sphere }\end{array}$

4 The supersphere as a conformal superspace $\quad 11$

$\begin{array}{lll}4.1 \text { Supertwistors } & 11\end{array}$

$\begin{array}{lll}4.2 & \text { The supersphere } & 13\end{array}$

$\begin{array}{lll}4.3 & \text { Bi-supertwistor realization } & 14\end{array}$

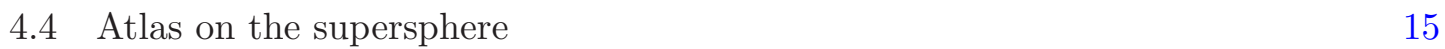

$\begin{array}{ll}\text { 4.5 Superconformal transformations } & 16\end{array}$

$\begin{array}{lll}4.6 & \text { Superconformal metric } & 18\end{array}$

$5 \mathcal{N}=2$ supersphere $\quad 19$

5.1 Superconformal transformations 20

$\begin{array}{ll}5.2 \text { Chiral subspace } & 21\end{array}$

5.3 Complexified supersphere 21

5.4 Superconformal inversion 22

6 Supercoset realizations of $\mathbb{E}^{3 \mid 4 n}$ and $S^{3 \mid 4 n} \quad 22$

6.1 The super-translation subalgebra of $\mathfrak{o s p}(2 n \mid 2,2)$ and $\mathbb{E}^{3 \mid 4 n} \quad 23$

6.2 The OSp $(2 n \mid 2) \times \operatorname{SU}(2)$ subalgebra of $\mathfrak{o s p}(2 n \mid 2,2)$ and $S^{3 \mid 4 n} \quad 24$

$6.3 S^{3 \mid 4}$ in the matrix realization of $\mathrm{SU}(2 \mid 1) \quad 27$

$\begin{array}{llr}7 & \text { Concluding comments and outlook } & 29\end{array}$

A Matrix realizations of $\operatorname{Sp}(2 n, \mathbb{R})$ and $\mathrm{SO}^{*}(2 n) \quad 31$

B Conformal spaces $\quad 32$

C Fibre bundles over the supersphere $\quad 33$ 


\section{Introduction}

Recently, there has been an interest (see, e.g., [1-3]) in superconformal field theories on a three-dimensional $(3 D)$ sphere, mostly motivated by the study of their quantum features with the use of localization techniques. In addition to the issues raised in [1-3] and related papers, it is also of interest to study correlation functions in superconformal field theories on $S^{3}$, and a superspace setting appears to be most suitable to address this goal. An $\mathcal{N}=2$ superspace formalism has been developed to describe $\mathcal{N}$-extended supersymmetric gauge theories on $S^{3}$ [4], but superconformal aspects of these and more general theories have not been studied in the Euclidean superspace framework so far. ${ }^{1}$

This paper is designed to be one of a series devoted to off-shell superconformal field theories on $S^{3}$ and is aimed at setting a geometric stage for their further study. We introduce a $2 n$-extended supersphere $S^{3 \mid 4 n}$, with $n=1,2, \ldots$, as a homogeneous space of the $3 D$ Euclidean superconformal group, $\operatorname{OSp}(2 n \mid 2,2)$, with the property that the bosonic body of $S^{3 \mid 4 n}$ is the three-sphere. ${ }^{2}$ Supertwistor and bi-supertwistor realizations of $S^{3 \mid 4 n}$ are derived. To some extent, these realizations are analogous to those of $3 \mathrm{D}$ and $4 \mathrm{D}$ compactified Minkowski superspaces $\overline{\mathbb{M}}^{3 \mid 2 \mathcal{N}}$ and $\overline{\mathbb{M}}^{4 \mid 4 \mathcal{N}}$, respectively, described in detail in [9-11]. However, the Euclidean case turns out to have new nontrivial features.

This paper is organized as follows. In section 2, we collect the main definitions concerning the $3 D$ Euclidean conformal and superconformal groups. In section 3, we describe the twistor and bitwistor realizations of $S^{3}$ as a warm-up for the subsequent supersymmetric constructions. The supertwistor and bi-supertwistor realizations of the $\mathcal{N}=2 n$ extended supersphere $S^{3 \mid 4 n}$ are presented in section 4 . The specific features of the $\mathcal{N}=2$ supersphere are analysed in section 5. Several supercoset realizations of $S^{3 \mid 4 n}$ and flat Euclidean superspace $\mathbb{E}^{3 \mid 4 n}$ are given in section 6 . The main body of the paper is accompanied by three appendices. In appendix $A$, we review two different matrix realizations for each of the groups $\operatorname{Sp}(2 n, \mathbb{R})$ and $\mathrm{SO}^{*}(2 n)$. Appendix B is a brief review of the Veblen-Dirac construction of pseudo-Euclidean conformal spaces $\overline{\mathbb{E}}^{s, t}$. In appendix $\mathrm{C}$, we sketch the construction of harmonic/projective extensions of $S^{3 \mid 4 n}$ by auxiliary bosonic variables.

\section{3D Euclidean (super)conformal groups}

In this section we define the conformal and superconformal groups in three Euclidean dimensions.

\footnotetext{
${ }^{1}$ The construction of $\mathcal{N}=2$ supersymmetric theories on $S^{3}[4]$ is similar to that of the off-shell $(2,0)$ supersymmetric field theories in $A d S_{3}$ given in [5]. In general, supersymmetric field theory in $A d S_{3}$ has so far been developed to a greater degree of completeness than its Euclidean $S^{3}$ counterpart. The supersymmetric extensions of $A d S_{3}$ were constructed in [5,6] and are known as the $(p, q)$ AdS superspaces, where $p \geq q$ are non-negative integers. For all types of $\mathcal{N}=3$ and $\mathcal{N}=4$ AdS supersymmetry, where $\mathcal{N}=p+q$, general off-shell supersymmetric field theories were constructed in a manifestly supersymmetric approach and also reformulated in $(2,0)$ AdS superspace [6-8].

${ }^{2}$ The supersphere $S^{3 \mid 4 n}$ has $4 n$ Grassmann-odd directions that are parametrized by $2 n$ two-component spinor coordinates.
} 


\subsection{The conformal group}

The conformal group of both the three-sphere $S^{3}$ and the Euclidean three-plane $\mathbb{E}^{3}$ is $\mathrm{SO}(4,1)$. The same group is also the isometry group of four-dimensional $(4 D)$ de Sitter space $d S_{4}$. Its connected component $\mathrm{SO}_{0}(4,1)$ is locally isomorphic ${ }^{3}$ to the $d S_{4}$ spin group $\operatorname{USp}(2,2)$ defined by

$$
\mathrm{USp}(2,2)=\operatorname{SU}(2,2) \bigcap \operatorname{Sp}(4, \mathbb{C}) d S
$$

Here $\mathrm{SU}(2,2)$ is a two to one covering group of the connected component $\mathrm{SO}_{0}(4,2)$ of the conformal group of four-dimensional Minkowski space $\mathbb{M}^{4}=\mathbb{E}^{3,1}$,

$$
\mathrm{SU}(2,2):=\left\{g \in \mathrm{SL}(4, \mathbb{C}), \quad g^{\dagger} I g=I, \quad I=\left(\begin{array}{cc}
\mathbb{1}_{2} & 0 \\
0 & -\mathbb{1}_{2}
\end{array}\right)\right\} .
$$

In the notation of appendix A, the matrix $I$ is $I_{2,2}$. The group $\operatorname{Sp}(4, \mathbb{C})_{d S}$ is simply the symplectic group $\operatorname{Sp}(4, \mathbb{C})$ in the following realization:

$$
\mathrm{Sp}(4, \mathbb{C})_{d S}:=\left\{g \in \mathrm{GL}(4, \mathbb{C}), \quad g^{\mathrm{T}} \Lambda g=\Lambda, \quad \Lambda=\left(\begin{array}{cc}
\sigma_{2} & 0 \\
0 & -\sigma_{2}
\end{array}\right)\right\},
$$

where $\sigma_{2}$ is the second Pauli matrix. The matrix $\Lambda$ satisfies the properties

$$
\Lambda^{\dagger}=-\Lambda^{\mathrm{T}}=\Lambda, \quad \Lambda^{2}=\mathbb{1}_{4} .
$$

It is instructive to compare the $d S_{4}$ spin group, USp $(2,2)$, with the one corresponding to $4 D$ anti-de Sitter space $A d S_{4}, \mathrm{Sp}(4, \mathbb{R})$. The latter is a two to one covering group of the connected component $\mathrm{SO}_{0}(3,2)$ of the isometry group of $A d S_{4}$. As shown in appendix A, this group can equivalently be realized as a subgroup of $\mathrm{SU}(2,2)$. This follows from the isomorphism

$$
\operatorname{Sp}(4, \mathbb{R}) \cong \operatorname{SU}(2,2) \bigcap \operatorname{Sp}(4, \mathbb{C})_{A d S},
$$

where $\operatorname{Sp}(4, \mathbb{C})_{A d S}$ stands for the symplectic group $\operatorname{Sp}(4, \mathbb{C})$ in the following realization:

$$
\mathrm{Sp}(4, \mathbb{C})_{A d S}:=\left\{g \in \mathrm{GL}(4, \mathbb{C}), \quad g^{\mathrm{T}} J g=J, \quad J=\left(\begin{array}{cc}
0 & \mathbb{1}_{2} \\
-\mathbb{1}_{2} & 0
\end{array}\right)\right\} .
$$

In the notation of appendix $\mathrm{A}$, the matrix $J$ is $J_{2,2}$.

As will be demonstrated in section 3 , the above matrix realization of $\operatorname{USp}(2,2)$ is most suitable to describe the global action of the superconformal group on the sphere $S^{3}$. However, in order to describe the conformal transformations in flat Euclidean space $\mathbb{E}^{3}$, a different matrix realization of $\operatorname{USp}(2,2)$ is more convenient. It is obtained from the original realization by applying the following similarity transformation:

$$
g \rightarrow \boldsymbol{g}=\Sigma g \Sigma^{-1}, \quad g \in \mathrm{USp}(2,2)
$$

\footnotetext{
${ }^{3}$ The group USp $(2,2)$ is a two to one covering group of $\mathrm{SO}_{0}(4,1)$.
} 
where we have introduced the orthogonal $4 \times 4$ matrix

$$
\Sigma=\frac{1}{\sqrt{2}}\left(\begin{array}{rr}
\mathbb{1}_{2} & -\mathbb{1}_{2} \\
\mathbb{1}_{2} & \mathbb{1}_{2}
\end{array}\right), \quad \Sigma^{\mathrm{T}} \Sigma=\mathbb{1}_{4} .
$$

In this realization, the elements of $\operatorname{USp}(2,2)$ obey the constraints

$$
\boldsymbol{g}^{\dagger} \boldsymbol{I} \boldsymbol{g}=\boldsymbol{I}, \quad \boldsymbol{g}^{\mathrm{T}} \boldsymbol{\Lambda} \boldsymbol{g}=\boldsymbol{\Lambda},
$$

where

$$
\boldsymbol{I}=\Sigma I \Sigma^{-1}=\left(\begin{array}{cc}
0 & \mathbb{1}_{2} \\
\mathbb{1}_{2} & 0
\end{array}\right), \quad \boldsymbol{\Lambda}=\Sigma \Lambda \Sigma^{-1}=\left(\begin{array}{cc}
0 & \sigma_{2} \\
\sigma_{2} & 0
\end{array}\right) .
$$

\subsection{The superconformal group}

$\mathcal{N}$-extended superconformal group in three Euclidean dimensions is

$$
\operatorname{OSp}(2 n \mid 2,2)=\operatorname{SU}(n, n \mid 2,2) \bigcap \operatorname{OSp}(2 n \mid 4 ; \mathbb{C}), \quad n=1,2, \ldots,
$$

with $\mathcal{N}=2 n$. It consists of $(2 n \mid 4) \times(2 n \mid 4)$ supermatrices (with $A, D$ bosonic blocks and $B, C$ fermionic ones)

$$
g=\left(\begin{array}{l|l}
A \mid B \\
\hline C & D
\end{array}\right)
$$

constrained by

$$
\begin{aligned}
& g^{\dagger} \Xi g=\Xi, \quad \Xi=\left(\begin{array}{c|c}
\Omega & 0 \\
\hline 0 & I
\end{array}\right), \quad \quad \Omega^{\dagger}=-\Omega^{\mathrm{T}}=\Omega, \quad \Omega^{2}=\mathbb{1}_{2 n}, \\
& g^{\mathrm{sT}} \Upsilon g=\Upsilon, \quad \Upsilon=\left(\begin{array}{c|c}
\mathbb{1}_{2 n} \mid & 0 \\
\hline 0 & \Lambda
\end{array}\right), \quad g^{\mathrm{sT}}=\left(\begin{array}{c|c}
A^{\mathrm{T}} \mid C^{\mathrm{T}} \\
\hline-B^{\mathrm{T}} \mid D^{\mathrm{T}}
\end{array}\right) .
\end{aligned}
$$

The bosonic subgroup of $\operatorname{OSp}(2 n \mid 2,2)$ is $\operatorname{SO}^{*}(2 n) \times \operatorname{USp}(2,2)$. Here we define the group $\mathrm{SO}^{*}(2 n)$ by

$$
\mathrm{SO}^{*}(2 n):=\left\{\mathfrak{U} \in \mathrm{GL}(2 n, \mathbb{C}), \quad \mathfrak{U}^{\mathrm{T}} \mathfrak{U}=\mathbb{1}_{2 n}, \quad \mathfrak{U}^{\dagger} \Omega \mathfrak{U}=\Omega\right\} .
$$

This definition of $\mathrm{SO}^{*}(2 n)$ is equivalent to the standard one given in appendix A, eq. (A.6). Indeed, it is always possible to choose $\Omega=\mathrm{i} J_{n, n}$ by applying a similarity transformation.

The above supermatrix realization of $\operatorname{OSp}(2 n \mid 2,2)$ is most suitable to consider the global action of the superconformal group on the supersphere $S^{3 \mid 4 n}$. However, in order to describe superconformal transformations in flat Euclidean superspace $\mathbb{E}^{3 \mid 4 n}$, a different supermatrix realization of $\operatorname{OSp}(2 n \mid 2,2)$ is more useful. It is obtained from the above realization by applying a similarity transformation

$$
g \rightarrow \boldsymbol{g}=\boldsymbol{\Sigma} g \boldsymbol{\Sigma}^{-1}, \quad g \in \operatorname{OSp}(2 n \mid 2,2)
$$

associated with the $(2 n \mid 4) \times(2 n \mid 4)$ supermatrix

$$
\boldsymbol{\Sigma}=\left(\begin{array}{c|c}
\mathbb{1}_{2 n} & 0 \\
\hline 0 & \Sigma
\end{array}\right)
$$


where $\Sigma$ is given by (2.8). In the new realization, the group elements of $\operatorname{OSp}(2 n \mid 2,2)$ obey the constraints

$$
\boldsymbol{g}^{\dagger} \boldsymbol{\Xi}=\boldsymbol{\Xi}, \quad \boldsymbol{g}^{\mathrm{sT}} \Upsilon \boldsymbol{g}=\Upsilon
$$

where

$$
\boldsymbol{\Xi}=\boldsymbol{\Sigma} \Xi \boldsymbol{\Sigma}^{-1}=\left(\begin{array}{c|cc}
\Omega & 0 & 0 \\
\hline 0 & 0 & \mathbb{1}_{2} \\
0 & \mathbb{1}_{2} & 0
\end{array}\right), \quad \boldsymbol{\Upsilon}=\boldsymbol{\Sigma} \Upsilon \boldsymbol{\Sigma}^{-1}=\left(\begin{array}{c|cc}
\mathbb{1}_{2 n} & 0 & 0 \\
\hline 0 & 0 & \sigma_{2} \\
0 & \sigma_{2} & 0
\end{array}\right)
$$

\subsection{The superconformal algebra}

Any element $\mathcal{L}$ of the superconformal algebra $\mathfrak{o s p}(2 n \mid 2,2)$ obeys the equations

$$
\begin{aligned}
\mathcal{L}^{\dagger} \Xi+\Xi \mathcal{L} & =0, \\
\mathcal{L}^{\mathrm{sT}} \Upsilon+\Upsilon \mathcal{L} & =0,
\end{aligned}
$$

which are the infinitesimal counterpart of (2.13). This gives a matrix realization of $\mathfrak{o} \mathfrak{s p}(2 n \mid 2,2)$. Alternatively, the superconformal algebra may be defined be specifying the corresponding (anti)commutation relations of its generators, and without resorting to any particular matrix realization.

The superalgebra osp $(2 n \mid 2,2)$ is formed by the generators $L_{\hat{a} \hat{b}}=-L_{\hat{a} \hat{b}}$ of $\operatorname{Sp}(2,2) \cong$ $\mathrm{SO}(4,1) / \mathbb{Z}_{2}(\hat{a}, \hat{b}=0,1,2,3,4)$, the generators $T^{i j}=-T^{j i}$ of SO*$(2 n)(i, j=1, \cdots 2 n)$ and $8 n$ supercharges $\mathcal{Q}_{\hat{\alpha}}^{i}(\hat{\alpha}=1,2,3,4) .{ }^{4}$ The defining anti-commutation relation of $\mathcal{Q}_{\hat{\alpha}}^{i}$ is (see e.g. [13])

$$
\left\{\mathcal{Q}_{\hat{\alpha}}^{i}, \mathcal{Q}_{\hat{\beta}}^{j}\right\}=\delta^{i j} \gamma_{\hat{\alpha} \hat{\beta}}^{\hat{a} \hat{b}} L_{\hat{a} \hat{b}}+C_{\hat{\alpha} \hat{\beta}} T^{i j}
$$

Here $C=\left(C_{\hat{\alpha} \hat{\beta}}\right)$ is a charge conjugation matrix, $C_{\hat{\alpha} \hat{\beta}}=-C_{\hat{\beta} \hat{\alpha}}=-C^{\hat{\alpha} \hat{\beta}}$, which we choose to be $C=\mathrm{i} \Lambda$ and which is used to raise and lower the spinor indices

$$
\mathcal{Q}^{i \hat{\alpha}}=C^{\hat{\alpha} \hat{\beta}} \mathcal{Q}_{\hat{\beta}}^{i}, \quad \mathcal{Q}_{\hat{\alpha}}^{i}=C_{\hat{\alpha} \hat{\beta}} \mathcal{Q}^{i \hat{\beta}} .
$$

The $D=5$ gamma-matrices $\gamma_{\hat{a}}=\left(\gamma_{\hat{a}}{ }_{\hat{\beta}}{ }_{\hat{\beta}}\right)$ obey the anti-commutation relation

$$
\left\{\gamma_{\hat{a}}, \gamma_{\hat{b}}\right\}=-2 \eta_{\hat{a} \hat{b}} \mathbb{1}_{4}
$$

where $\eta_{\hat{a} \hat{b}}=\operatorname{diag}(-,+,+,+,+)$ is the Minkowski metric. These matrices have the standard properties

$$
\gamma_{\hat{a}}^{\dagger}=\gamma_{0} \gamma_{\hat{a}} \gamma_{0}, \quad\left(\gamma_{\hat{a}}\right)^{\mathrm{T}}=C \gamma_{\hat{a}} C^{-1},
$$

which imply that $\gamma_{\hat{\alpha} \hat{\beta}}^{\hat{a}} \equiv\left(C \gamma^{\hat{a}}\right)_{\hat{\alpha} \hat{\beta}}=-\left(C \gamma^{\hat{a}}\right)_{\hat{\beta} \hat{\alpha}}$ are antisymmetric and $\gamma_{\hat{\alpha} \hat{\beta}}^{\hat{a} \hat{b}} \equiv\left(C \gamma^{\hat{a} \hat{b}}\right)_{\hat{\alpha} \hat{\beta}}=$ $\left(C \gamma^{\hat{a} \hat{b}}\right)_{\hat{\beta} \hat{\alpha}}$ are symmetric matrices. A convenient representation for $\gamma_{\hat{a}}$ is

$$
\gamma_{0}=\left(\begin{array}{cc}
\mathbb{1}_{2} & 0 \\
0 & -\mathbb{1}_{2}
\end{array}\right)=I, \quad \gamma_{a}=\left(\begin{array}{cc}
0 & \mathrm{i} \sigma_{a} \\
\mathrm{i} \sigma_{a} & 0
\end{array}\right), \quad \gamma_{4}=\left(\begin{array}{cc}
0 & \mathbb{1}_{2} \\
-\mathbb{1}_{2} & 0
\end{array}\right) .
$$

\footnotetext{
${ }^{4}$ Note that $\mathfrak{s o}(4,1)$ generates the isometries of $d S_{4}$ space, and the superalgebra $\mathfrak{o s p}(2 n \mid 2,2)$ is a unique superextension of this algebra (see e.g. $[12,13])$. $4 D$ supergravity theories based on the $n=1 d S_{4}$ superalgebra were shown $[14,15]$ to contain ghosts.
} 
The superconformal generators $\mathcal{Q}^{i}$ are symplectic-Majorana spinors

$$
\mathcal{Q}^{i}=\mathrm{i} \Omega^{i j} C\left(\overline{\mathcal{Q}}^{j}\right)^{T}, \quad \overline{\mathcal{Q}}^{i}=\left(\mathcal{Q}^{i}\right)^{\dagger} \gamma_{0},
$$

where $\Omega^{i j}=-\Omega^{j i}$ is an $\mathrm{SO}^{*}(2 n)$ invariant symplectic form defined in (2.13a). Note that the $\mathrm{SO}^{*}(2 n)$ indices are raised and lowered by the orthogonal unit metric $\delta^{i j}$.

Upon splitting the $D=5$ indices $\hat{a}, \hat{b}$ into Euclidean $D=3$ indices $a, b=1,2,3$ and the rest, for instance $\hat{a}=(0, a, 4)$, we get the following $D=3$ adapted form of the $\operatorname{OSp}(2 n \mid 2,2)$ algebra

$$
\left\{\mathcal{Q}^{i}, \mathcal{Q}^{j}\right\}=\delta^{i j}\left(\gamma^{a b} L_{a b}+2 \gamma^{a} \gamma^{0} L_{a 0}+2 \gamma^{a} \gamma^{4} L_{a 4}+2 \gamma^{4} \gamma^{0} L_{40}\right)+C T^{i j},
$$

where $L_{a b}$ generate an $\mathrm{SO}(3) \cong \mathrm{SU}(2) / \mathbb{Z}_{2}$ subgroup of the conformal group $\mathrm{SO}(4,1)$ and $L_{a 4}$ can be associated with the operators that generate translations in $S^{3}$, i.e. $L_{a b}$ and $L_{a 4}$ form the $\mathrm{SO}(4) \cong(\mathrm{SU}(2) \times \mathrm{SU}(2)) / \mathbb{Z}_{2}$ isometry of $S^{3}$, while $K_{a}=\mathrm{i}\left(L_{a 0}-L_{a 4}\right)$ and $L_{40}$ generate, respectively, the conformal boosts and dilatations of $S^{3}$.

The translations in a flat Euclidean $D=3$ space are generated by $P_{a}=\mathrm{i}\left(L_{a 0}+L_{a 4}\right)$, while the flat space conformal boosts are generated by $K_{a}=\mathrm{i}\left(L_{a 0}-L_{a 4}\right)$. Note that $\left[P_{a}, P_{b}\right]=0=\left[K_{a}, K_{b}\right]$

\section{The three-sphere as a conformal space}

In this section we present twistor and bitwistor realizations for the three-sphere. ${ }^{5}$

\subsection{Twistor realization of the three-sphere}

Introduce two USp $(2,2)$ invariant inner products on $\mathbb{C}^{4}$ :

$$
\begin{aligned}
\langle S \mid T\rangle_{I} & :=S^{\dagger} I T=\overline{S_{\hat{\alpha}}} I^{\hat{\alpha} \hat{\beta}} T_{\hat{\beta}}, \\
\langle S \mid T\rangle_{\Lambda} & :=S^{\mathrm{T}} \Lambda T=S_{\hat{\alpha}} \Lambda^{\hat{\alpha} \hat{\beta}} T_{\hat{\beta}},
\end{aligned}
$$

for any $T, S \in \mathbb{C}^{4}$. We will refer to this space as twistor space, and its elements will be called twistors. A twistor is viewed as a column vector

$$
T=\left(T_{\hat{\alpha}}\right)=\left(\begin{array}{c}
f_{\alpha} \\
g_{\beta}
\end{array}\right),
$$

with the two-component spinors $f_{\alpha}$ and $g_{\beta}$ being complex.

Consider the space of all two-planes in $\mathbb{C}^{4}$ known as the Grassmannian $G_{2,4}(\mathbb{C})$. Any two-plane is determined by its basis, i.e. by two linearly independent twistors $T^{\mu}$, with $\mu=1,2$. Such a basis $\left\{T^{\mu}\right\}$ is defined only modulo the equivalence relation

$$
\left\{T^{\mu}\right\} \sim\left\{\tilde{T}^{\mu}\right\}, \quad \tilde{T}^{\mu}=T^{\nu} R_{\nu}^{\mu}, \quad R \in \mathrm{GL}(2, \mathbb{C}) .
$$

\footnotetext{
${ }^{5}$ The twistor and bitwistor realizations for $4 D$ conformal spaces $\overline{\mathbb{E}}^{3,1} \equiv \overline{\mathbb{M}}^{4}$ and $\overline{\mathbb{E}}^{4,0} \equiv S^{4}$ (see appendix B for more details) were given by Veblen in 1933 [16] who used the Plücker-Klein correspondence. He introduced the term "spin-space" for what nowadays is known as "twistor space." Dirac learnt his realization [17] of the conformal space $\overline{\mathrm{M}}^{4}$, which is reviewed in appendix B, from Veblen as acknowledged in [17].
} 
Equivalently, the Grassmannian $G_{2,4}(\mathbb{C})$ can be thought of as consisting of all $4 \times 2$ complex matrices of rank two,

$$
\left(T^{1} T^{2}\right)=\left(\begin{array}{c}
F \\
G
\end{array}\right),
$$

where the $2 \times 2$ matrices $F$ and $G$ are defined modulo the equivalence relation

$$
\left(\begin{array}{l}
F \\
G
\end{array}\right) \sim\left(\begin{array}{l}
F R \\
G R
\end{array}\right), \quad R \in \mathrm{GL}(2, \mathbb{C}) .
$$

Let $\mathfrak{S}$ denote the subspace of $G_{2,4}(\mathbb{C})$ consisting of all two-planes in $\mathbb{C}^{4}$ that are null with respects to the two inners products (3.1). For any two-plane belonging to $\mathfrak{S}$, it holds that

$$
\left\langle T^{\mu} \mid T^{\nu}\right\rangle_{I}=0, \quad\left\langle T^{\mu} \mid T^{\nu}\right\rangle_{\Lambda}=0, \quad \mu, \nu=1,2
$$

or, equivalently,

$$
\begin{aligned}
F^{\dagger} F-G^{\dagger} G & =0, \\
F^{\mathrm{T}} \sigma_{2} F-G^{\mathrm{T}} \sigma_{2} G & =0 .
\end{aligned}
$$

It is known that the space of all two-planes in $\mathbb{C}^{4}$ under the null condition (3.7a) is compactified 4D Minkowski space, $\overline{\mathbb{M}}^{4}=\left(S^{3} \times S^{1}\right) / \mathbb{Z}_{2}$, see e.g. [10]. As shown in [10], the conditions that the $4 \times 2$ matrix (3.4) has rank two and obeys (3.7a) imply that

$$
\operatorname{det} F \neq 0 \quad \text { and } \quad \operatorname{det} G \neq 0 \text {. }
$$

The equivalence relation (3.5) tells us that

$$
\left(\begin{array}{l}
F \\
G
\end{array}\right) \sim\left(\begin{array}{c}
h \\
\mathbb{1}_{2}
\end{array}\right) .
$$

Now the conditions (3.7a) and (3.7b) imply, respectively,

$$
\begin{aligned}
& h^{\dagger} h=\mathbb{1}_{2} \quad \Longrightarrow \quad h \in \mathrm{U}(2) ; \\
& h^{\mathrm{T}} \sigma_{2} h=\sigma_{2} \quad \Longrightarrow \quad \operatorname{det} h=1 \text {. }
\end{aligned}
$$

We conclude that $\mathfrak{S}$ may be identified with the group manifold $\mathrm{SU}(2)=S^{3}$.

Given a group element

$$
g=\left(g_{\hat{\alpha}}^{\hat{\beta}}\right)=\left(\begin{array}{ll}
A & B \\
C & D
\end{array}\right) \in \operatorname{USp}(2,2),
$$

with $A, B, C$ and $D$ some $2 \times 2$ matrices, its action on $S^{3}$ is a fractional linear transformation

$$
h \rightarrow h^{\prime}=(A h+B)(C h+D)^{-1} .
$$




\subsection{Real structure}

Twistors transform in the defining representation of $\operatorname{USp}(2,2)$. Given a group element of USp $(2,2)$, eq. (3.11), it acts on twistor space as

$$
T_{\hat{\alpha}} \rightarrow T_{\hat{\alpha}}^{\prime}=g_{\hat{\alpha}}^{\hat{\beta}} T_{\hat{\beta}} .
$$

Let us also consider the dual of twistor space. Its elements are complex row vectors $V=\left(V^{\hat{\alpha}}\right)=\left(v^{\alpha}, w^{\beta}\right)$ possessing the $\operatorname{USp}(2,2)$ transformation law

$$
V^{\hat{\alpha}} \rightarrow V^{\prime \hat{\alpha}}=V^{\hat{\beta}}\left(g^{-1}\right)_{\hat{\beta}}^{\hat{\alpha}} .
$$

Since both inners products $(3.1)$ are $\operatorname{USp}(2,2)$ invariant, we conclude that $S^{\dagger} I$ and $S^{\mathrm{T}} \Lambda$ are dual twistors for any twistor $S$. The dual of $T_{\hat{\alpha}}$ is defined to be

$$
\bar{T}^{\hat{\alpha}}:=\bar{T}_{\hat{\beta}} I^{\hat{\beta} \hat{\alpha}}=I^{\hat{\alpha} \hat{\beta}} \overline{T_{\hat{\beta}}} .
$$

We also point out that $\Lambda^{\hat{\alpha} \hat{\beta}}$ is an invariant tensor of USp(2,2), and so is its inverse $\Lambda^{-1}=$ $\left(\Lambda_{\hat{\alpha} \hat{\beta}}\right)$. As a result, we can define a one-to-one anti-linear map of twistor space onto itself,

$$
\star: T_{\hat{\alpha}} \rightarrow \Lambda_{\hat{\alpha} \hat{\beta}} I^{\hat{\beta} \hat{\gamma}} \overline{T_{\hat{\gamma}}},
$$

for any twistor $T$. This map induces a well defined transformation on the Grassmannian $G_{2,4}(\mathbb{C})$,

$$
\mathcal{P}=\left(\begin{array}{c}
F \\
G
\end{array}\right) \rightarrow \star \mathcal{P}=\Lambda^{-1} I\left(\begin{array}{c}
\bar{F} \\
\bar{G}
\end{array}\right) .
$$

This transformation is well defined in the sense that any two equivalent $4 \times 2$ matrices $\mathcal{P}$ and $\mathcal{P} R$, with $R \in \mathrm{GL}(2, \mathbb{C})$, are mapped into equivalent ones, $\star \mathcal{P}$ and $(\star \mathcal{P}) \bar{R}$, where $\bar{R}$ denotes the complex conjugate of $R$.

The map (3.16) is characterized by the property $\star \star=-\mathbb{1}_{4}$, and therefore it cannot be used to define a complex conjugation on twistor space. ${ }^{6}$ However, the map (3.17) may be seen to define an involution on the space of all two planes in twistor space, $\star \star x=i d$. Now consider any null two-plane defined by the relations (3.9) and (3.10). It is straightforward to show that this two-plane is real with respect to the involution introduced.

\subsection{Bitwistor realization}

Let $T_{\hat{\alpha}}{ }^{\mu}$ be two linearly independent twistors that form a basis of a two-plane in $\mathbb{C}^{4}$. We can associate with them a bitwistor

$$
X_{\hat{\alpha} \hat{\beta}}:=T_{\hat{\alpha}}^{\mu} T_{\hat{\beta}}^{\nu} \varepsilon_{\mu \nu}=-X_{\hat{\beta} \hat{\alpha}}, \quad \varepsilon_{\mu \nu}=-\varepsilon_{\nu \mu}, \quad \varepsilon_{12}=-1
$$

and its dual

$$
\bar{X}^{\hat{\alpha} \hat{\beta}}:=\varepsilon_{\mu \nu} \bar{T}^{\mu \hat{\alpha}} \bar{T}^{\nu \hat{\beta}}=I^{\hat{\alpha} \hat{\gamma}} I^{\hat{\beta} \hat{\delta}} \overline{X_{\hat{\gamma} \hat{\delta}}}, \quad \bar{T}^{\mu \hat{\alpha}}:=\overline{T^{\hat{\alpha} \mu}},
$$

\footnotetext{
${ }^{6}$ The map (3.16) does not allow us to define real lines in the space $\mathbb{C} P^{3}$ of lines in twistor space.
} 
with $I=\left(I^{\hat{\alpha} \hat{\beta}}\right)$. In terms of $X_{\hat{\alpha} \hat{\beta}}$, the equivalence relation (3.3) turns into

$$
X_{\hat{\alpha} \hat{\beta}} \sim c X_{\hat{\alpha} \hat{\beta}}, \quad c \in \mathbb{C} \backslash\{0\} .
$$

In the case that the twistors $T_{\hat{\alpha}}{ }^{\mu}$ describe a null two-plane, eq. (3.6), the corresponding bitwistor $X_{\hat{\alpha} \hat{\beta}}$ has the following algebraic properties:

$$
\begin{aligned}
X_{[\hat{\alpha} \hat{\beta}} X_{\hat{\gamma} \hat{\delta}]} & =0, \\
\Lambda^{\hat{\beta} \hat{\alpha}} X_{\hat{\alpha} \hat{\beta}} & =0, \\
\bar{X}^{\hat{\alpha} \hat{\gamma}} X_{\hat{\gamma} \hat{\beta}} & =0 .
\end{aligned}
$$

As shown in subsection 3.2, all null two-planes are real with respect to the anti-linear map (3.16). Recast in terms of $X_{\hat{\alpha} \hat{\beta}}$, this property means the following:

$$
\Lambda_{\hat{\alpha} \hat{\gamma}} \Lambda_{\hat{\beta} \hat{\delta}} \bar{X}^{\hat{\gamma} \hat{\delta}} \sim X_{\hat{\alpha} \hat{\beta}}
$$

The above discussion naturally leads us to an alternative realization of $S^{3}$ as the space of non-zero bitwistors $X_{\hat{\alpha} \hat{\beta}}$ subject to the constraints (3.21) and defined modulo the equivalence relation (3.20). Equivalence of this bitwistor realization of $S^{3}$ to the twistor one given in subsection 3.1 can be proved in complete analogy to the case of compactified 3D Minkowski space [11]. Constraint (3.21a) means that $X_{\hat{\alpha} \hat{\beta}}$ is decomposable, eq. (3.18). The constraints (3.21b) and (3.21c) prove to imply the null conditions (3.6).

The bitwistor realization is intimately related to the Veblen-Dirac realization of $S^{3}$, see appendix B. To see this, using the gamma-matrices (2.23), we introduce a null five-vector

$$
X_{\hat{a}}:=\gamma_{\hat{a}}^{\hat{\alpha} \hat{\beta}} X_{\hat{\alpha} \hat{\beta}}, \quad \eta_{\hat{a} \hat{b}} X^{\hat{a}} X^{\hat{b}}=0 .
$$

This vector is defined up to re-scalings and, due to (3.22), may be chosen to be real. As a result, we arrive at the realization of $S^{3}$ described in appendix B.

\subsection{Atlas on the three-sphere}

Let us switch to a new parametrization of the group $\operatorname{USp}(2,2)$ that is more convenient for describing the conformal transformations in $\mathbb{E}^{3}$. This parametrization is obtained by applying the similarity transformation

$$
\begin{array}{rlrl}
g & \rightarrow \boldsymbol{g}=\Sigma g \Sigma^{-1}, & g & \in \mathrm{USp}(2,2), \\
T & \rightarrow \boldsymbol{T}=\Sigma T, & T \in \mathbb{C}^{4},
\end{array}
$$

with the matrix $\Sigma$ given by (2.8). The matrices $I$ and $\Lambda$, which determine the inner products (3.1), turn into those given by (2.10), while the two-plane turns into

$$
\mathcal{P}=\left(\begin{array}{c}
h \\
\mathbb{1}_{2}
\end{array}\right) \rightarrow \mathcal{P}=\frac{1}{\sqrt{2}}\left(\begin{array}{c}
h-\mathbb{1}_{2} \\
h+\mathbb{1}_{2}
\end{array}\right) .
$$

The equations $\operatorname{det}\left(h+\mathbb{1}_{2}\right)=0$ and $\operatorname{det}\left(h-\mathbb{1}_{2}\right)=0$, with $h \in \mathrm{SU}(2)$, have unique solutions $h=-\mathbb{1}_{2}$ and $h=\mathbb{1}_{2}$, respectively. As a result, the sphere $S^{3}$ can be covered by two open 
charts, $S^{3}=U_{\mathrm{N}} \cup U_{\mathrm{S}}$. The north chart $U_{\mathrm{N}}$ is defined to consist of all null two-planes for which $\operatorname{det}\left(h+\mathbb{1}_{2}\right) \neq 0$. In this chart

$$
\left(\begin{array}{c}
h-\mathbb{1}_{2} \\
h+\mathbb{1}_{2}
\end{array}\right) \sim\left(\begin{array}{c}
\mathrm{i} x_{\mathrm{N}} \\
\mathbb{1}_{2}
\end{array}\right), \quad \text { i } x_{\mathrm{N}}=\frac{h-\mathbb{1}_{2}}{h+\mathbb{1}_{2}} .
$$

Similarly, the south chart $U_{\mathrm{S}}$ is spanned by all null two-planes with $\operatorname{det}\left(h-\mathbb{1}_{2}\right) \neq 0$. In this chart

$$
\left(\begin{array}{l}
h-\mathbb{1}_{2} \\
h+\mathbb{1}_{2}
\end{array}\right) \sim\left(\begin{array}{c}
\mathbb{1}_{2} \\
\mathrm{i} x_{\mathrm{S}}
\end{array}\right), \quad \text { i } x_{\mathrm{S}}=\frac{h+\mathbb{1}_{2}}{h-\mathbb{1}_{2}} .
$$

In the overlap of the two charts, $U_{\mathrm{N}} \cap U_{\mathrm{S}}$, we have the transition function

$$
x_{\mathrm{S}}=-x_{\mathrm{N}}^{-1} \text {. }
$$

In the remainder of this subsection, we work in the north chart and denote the $2 \times 2$ matrix $x_{\mathrm{N}}$ simply by $x$. The null conditions (3.10) imply that the matrix $x$ is constrained by

$$
x^{\dagger}=x, \quad x^{\mathrm{T}}=-\sigma_{2} x \sigma_{2} \quad \Longrightarrow \quad x=\vec{x} \cdot \vec{\sigma}, \quad \vec{x} \in \mathbb{R}^{3} .
$$

Thus we may think of $S^{3}$ as $\mathbb{R}^{3} \cup\left\{\infty_{\mathrm{N}}\right\}$, where $\mathbb{R}^{3}$ is identified with $U_{\mathrm{N}}$ and the point $\infty_{\mathrm{N}}$ is identified with the null two-plane

$$
\mathcal{P}_{\infty_{N}}=\left(\begin{array}{c}
\mathbb{1}_{2} \\
0
\end{array}\right)
$$

which corresponds to the origin of the coordinate chart $U_{\mathrm{S}}$.

In the new parametrization introduced, the conformal group USp $(2,2)$ consists of all $4 \times 4$ matrices $g$ of the form:

$$
\boldsymbol{g}=\left(\begin{array}{cc}
\mathcal{A} & \mathcal{B} \\
\mathcal{C} & \mathcal{D}
\end{array}\right), \quad \boldsymbol{g}^{\dagger} \boldsymbol{I} \boldsymbol{g}=\boldsymbol{I}, \quad \boldsymbol{g}^{\mathrm{T}} \boldsymbol{\Lambda} \boldsymbol{g}=\boldsymbol{\Lambda}
$$

Given such a group element, $\boldsymbol{g} \in \mathbf{U S p}(2,2)$, it generates the following transformation on $S^{3}$ :

$$
\mathrm{i} x \rightarrow \mathrm{i} x^{\prime}=(\mathrm{i} \mathcal{A} x+\mathcal{B})(\mathrm{i} \mathcal{C} x+\mathcal{D})^{-1} .
$$

The isotropy group of the point $\infty_{\mathrm{N}}$ consists of all matrices of the form:

$$
\left(\begin{array}{cc}
\mathbb{1}_{2} & \mathrm{ib} \\
0 & \mathbb{1}_{2}
\end{array}\right)\left(\begin{array}{cc}
\mathrm{e}^{\frac{1}{2} \lambda} \mathbb{1}_{2} & 0 \\
0 & \mathrm{e}^{-\frac{1}{2} \lambda} \mathbb{1}_{2}
\end{array}\right)\left(\begin{array}{cc}
\mathfrak{R} & 0 \\
0 & \mathfrak{R}
\end{array}\right), \quad \lambda \in \mathbb{R}, \quad \mathfrak{R} \in \operatorname{SU}(2),
$$

where we have denoted $\mathfrak{b}:=\vec{b} \cdot \vec{\sigma}, \vec{b} \in \mathbb{R}^{3}$. The parameters $\vec{b}, \lambda$ and $\mathfrak{R}$ describe, respectively, a translation, a dilatation and a rotation of Euclidean three-plane $\mathbb{E}^{3}$. Transformations (3.33) with $\lambda=0$ span the connected isometry group of $\mathbb{E}^{3}$, ISO $\mathrm{IS}_{0}(3)$.

The origin of $U_{\mathrm{N}}, x=0$, is the infinitely separated point $\infty_{\mathrm{S}}$ for $U_{\mathrm{S}}$. The isotropy group of this point consists of all matrices of the form:

$$
\left(\begin{array}{cc}
\mathbb{1}_{2} & 0 \\
\mathrm{ic} & \mathbb{1}_{2}
\end{array}\right)\left(\begin{array}{cc}
\mathrm{e}^{\frac{1}{2} \lambda} \mathbb{1}_{2} & 0 \\
0 & \mathrm{e}^{-\frac{1}{2} \lambda} \mathbb{1}_{2}
\end{array}\right)\left(\begin{array}{cc}
\mathfrak{R} & 0 \\
0 & \mathfrak{R}
\end{array}\right), \quad \lambda \in \mathbb{R}, \quad \mathfrak{R} \in \mathrm{SU}(2),
$$

with $\mathfrak{c}:=\vec{c} \cdot \vec{\sigma}, \vec{c} \in \mathbb{R}^{3}$. As follows from (3.32), the parameter $\vec{c}$ generates a special conformal transformation of $\mathbb{E}^{3}$. 


\section{The supersphere as a conformal superspace}

In this section we introduce a $2 n$-extended supersphere $S^{3 \mid 4 n}$ as a homogeneous space for the superconformal group $\operatorname{OSp}(2 n \mid 2,2)$. For this we develop supertwistor and bi-supertwistor realizations for the supersphere. ${ }^{7}$

\subsection{Supertwistors}

The supergroup $\operatorname{OSp}(2 n \mid 2,2)$ naturally acts on the space of even supertwistors and also on the space of odd supertwistors. An arbitrary supertwistor looks like

$$
T=\left(T_{A}\right)=\left(\begin{array}{c}
T_{i} \\
T_{\hat{\alpha}}
\end{array}\right), \quad i=1, \ldots, 2 n .
$$

In the case of even supertwistors, $T_{i}$ is fermionic and $T_{\hat{\alpha}}$ is bosonic. In the case of odd supertwistors, $T_{i}$ is bosonic and $T_{\hat{\alpha}}$ is fermionic. We introduce the parity function $\varepsilon(T)$ defined as: $\varepsilon(T)=0$ if $T$ is even, and $\varepsilon(T)=1$ if $T$ is odd. We also define

$$
\varepsilon_{A}=\left\{\begin{array}{cc}
1 & A=i \\
0 & A=\hat{\alpha}
\end{array} .\right.
$$

Then the above definition can be rewritten as

$$
\varepsilon\left(T_{A}\right)=\varepsilon(T)+\varepsilon_{A} \quad(\bmod 2) .
$$

Even and odd supertwistors are called pure. ${ }^{8}$ The space of even supertwistors may be identified with $\mathbb{C}^{4 \mid 2 n}$.

Supertwistors transform in the defining representation of $\operatorname{OSp}(2 n \mid 2,2)$,

$$
T \rightarrow T^{\prime}=g T, \quad g \in \operatorname{OSp}(2 n \mid 2,2) .
$$

This transformation law implies that the supergroup $\operatorname{OSp}(2 n \mid 2,2)$ defined by $(2.11)-(2.13)$ leaves invariant two inner products

$$
\begin{aligned}
\langle S \mid T\rangle_{\Xi} & :=S^{\dagger} \Xi T=\overline{S_{A}} \Xi^{A B} T_{B}, \\
\langle S \mid T\rangle_{\Upsilon} & :=(-1)^{\varepsilon_{A}+\varepsilon(S) \cdot \varepsilon_{A}} S_{A} \Upsilon^{A B} T_{B},
\end{aligned}
$$

for arbitrary pure supertwistors $S$ and $T$. These inner products have the following fundamental properties:

$$
\overline{\left\langle T_{1} \mid T_{2}\right\rangle_{\Xi}}=\left\langle T_{2} \mid T_{1}\right\rangle_{\Xi}
$$

\footnotetext{
${ }^{7}$ The concept of supertwistors was introduced by Ferber [18] within the framework of $4 D$ conformal supersymmetry. The supertwistor realization for compactified $4 D \mathcal{N}$-extended Minkowski superspace $\overline{\mathbb{M}}^{4 \mid 4 \mathcal{N}}$ was developed by Manin [19, 20] and also Kotrla and Niederle [21]. The bi-supertwistor realization for the same superspace was first considered by Siegel [22, 23], although it naturally follows from Manin's construction $[19,20]$. See $[10,11]$ for modern descriptions of these realizlations.

${ }^{8}$ This terminology is natural within the framework of supervector spaces $[24,25]$ and should not be confused with Cartan's pure spinors [26].
} 


$$
\left\langle T_{1} \mid T_{2}\right\rangle_{\Upsilon}=-(-1)^{\varepsilon_{1} \varepsilon_{2}}\left\langle T_{2} \mid T_{1}\right\rangle_{\Upsilon}
$$

for arbitrary pure supertwistors $T_{1}$ and $T_{2}$.

A dual supertwistor

$$
Z=\left(Z^{A}\right)=\left(Z^{i}, Z^{\hat{\alpha}}\right), \quad i=1, \ldots, 2 n
$$

transforms under $\operatorname{OSp}(2 n \mid 2,2)$ such that $Z^{A} T_{A}$ is invariant for any supertwistor $T$,

$$
Z \rightarrow Z^{\prime}=Z g^{-1}, \quad g \in \mathrm{OSp}(2 n \mid 2,2)
$$

A dual supertwistor $Z$ is even (odd) if $Z^{A} T_{A}$ is a $c$-number for any even (odd) supertwistor $T$.

Invariance of the inner product (4.4b) under $\operatorname{OSp}(2 n \mid 2,2)$ tells us that

$$
Z^{A}:=-(1)^{\varepsilon_{B}+\varepsilon(S) \varepsilon_{B}} S_{B} \Upsilon^{B A}=(-1)^{\varepsilon(S) \varepsilon_{A}} \Upsilon^{A B} S_{B}
$$

is a pure dual supertwistor. Conversely, given a pure dual supertwistor $Z^{A}$, the following object

$$
S_{A}:=(-1)^{\varepsilon(Z) \varepsilon_{B}}\left(\Upsilon^{-1}\right)_{A B} Z^{B}
$$

is a pure supertwistor. We emphasize that $\Upsilon^{A B}$ is an invariant tensor of the superconformal group,

$$
\left(g^{\mathrm{sT}}\right)_{C}^{A} \Upsilon^{C D} g_{D}{ }^{B}=\Upsilon^{A B}, \quad\left(g^{\mathrm{sT}}\right)^{A}{ }_{B}=(-1)^{\varepsilon_{A} \varepsilon_{B}+\varepsilon_{B}} g_{B}{ }^{A}
$$

for any group element $g \in \operatorname{OSp}(2 n \mid 2,2)$.

Since the inner product (4.4a) is invariant under $\operatorname{OSp}(2 n \mid 2,2) \subset$, we observe that

$$
\bar{S}^{A}:=\overline{S_{B}} \Xi^{B A}
$$

is a dual supertwistor, for any pure supertwistor $S_{A} \cdot{ }^{9}$ In conjunction with our previous result (4.9), this implies the existence of a one-to-one map of supertwistor space onto itself defined by

$$
\star: S_{A} \rightarrow(\star S)_{A}:=(-1)^{\varepsilon_{C}+\varepsilon(S) \varepsilon_{C}}\left(\Upsilon^{-1}\right)_{A B} \Xi^{B C} \overline{S_{C}},
$$

for any pure supertwistor $S_{A}$. This map is characterized by the property

$$
\star \star=-\mathbb{1}_{2 n \mid 4},
$$

which follows from the observations that the matrices $\Omega$ and $\Lambda I$ (i) are purely imaginary; and (ii) fulfill the identities $\Omega^{2}=\mathbb{1}_{2 n}$ and $(\Lambda I)^{2}=\mathbb{1}_{4}$.

\footnotetext{
${ }^{9}$ Eq. (4.4a) can be rewritten in the form $\langle S \mid T\rangle_{\Xi}=\bar{S}^{A} T_{A}$.
} 


\subsection{The supersphere}

We define a $2 n$-extended supersphere $S^{3 \mid 4 n}$ to be the space of all null and real two-planes in the space of even supertwistors $\mathbb{C}^{4 \mid 2 n}$. In general, any two-plane in $\mathbb{C}^{4 \mid 2 n}$ is generated by two supertwistors $T^{\mu}$ such that their bodies are linearly independent. Equivalently, it may be described by a rank-two $(2 n \mid 4) \times 2$ supermatrix

$$
\left(T^{\mu}\right)=\left(\begin{array}{c}
\Theta \\
F \\
G
\end{array}\right), \quad \mu=1,2,
$$

which is defined modulo the equivalence relation

$$
\left(\begin{array}{l}
\Theta \\
F \\
G
\end{array}\right) \sim\left(\begin{array}{c}
\frac{\Theta R}{F R} \\
G R
\end{array}\right), \quad R \in \mathrm{GL}(2, \mathbb{C})
$$

Here $\Theta$ is a $2 n \times 2$ fermionic matrix, and $F$ and $G$ are $2 \times 2$ bosonic matrices. The two-planes belonging to $S^{3 \mid 4 n}$ are required to be (i) null with respect to the two inner products (4.4); and (ii) real with respect to the star-map (4.12) modulo the equivalence relation (4.15). The null conditions are

$$
\begin{aligned}
\Theta^{\dagger} \Omega \Theta+F^{\dagger} F-G^{\dagger} G & =0 ; \\
-\Theta^{\mathrm{T}} \Theta+F^{\mathrm{T}} \sigma_{2} F-G^{\mathrm{T}} \sigma_{2} G & =0 .
\end{aligned}
$$

As in the bosonic case, the first null condition implies that $\operatorname{det} F \neq 0$ and $\operatorname{det} G \neq 0$. As a result, the null two-plane can equivalently be described by a supermatrix

$$
\mathcal{P}=\left(\begin{array}{c}
\Theta \\
\hline \boldsymbol{h} \\
\mathbb{1}_{2}
\end{array}\right)=\left(\begin{array}{c}
\Theta_{i}^{\beta} \\
\boldsymbol{h}_{\alpha}{ }^{\beta} \\
\delta_{\gamma}{ }^{\beta}
\end{array}\right),
$$

where the null conditions (4.16) now read

$$
\begin{aligned}
\Theta^{\dagger} \Omega \Theta+\boldsymbol{h}^{\dagger} \boldsymbol{h} & =\mathbb{1}_{2}, \\
-\Theta^{\mathrm{T}} \Theta+\boldsymbol{h}^{\mathrm{T}} \sigma_{2} \boldsymbol{h} & =\sigma_{2} .
\end{aligned}
$$

The condition that the two-plane (4.17) is real under (4.12) amounts to

$$
\begin{aligned}
& \bar{\Theta}=-\Omega \Theta \sigma_{2}, \\
& \overline{\boldsymbol{h}}=\sigma_{2} \boldsymbol{h} \sigma_{2} .
\end{aligned}
$$

Eq. (4.19a) is a pseudo-Majorana condition. 


\subsection{Bi-supertwistor realization}

The bitwistor realization of the three-sphere given in subsection 3.3 can naturally be generalized to the case of the supersphere.

Let $T_{A}{ }^{\mu}$ be two linearly independent even supertwistors belonging to a two-plane in $\mathbb{C}^{4 \mid 2 n}$. We can associate with them a bi-supertwistor

$$
X_{A B}:=T_{A}{ }^{\mu} T_{B}{ }^{\nu} \varepsilon_{\mu \nu}=-(-1)^{\varepsilon_{A} \varepsilon_{B}} X_{B A} .
$$

The equivalence relation (4.15) turns into

$$
X_{A B} \sim c X_{A B}, \quad c \in \mathbb{C} \backslash\{0\} .
$$

Using the dual supertwistors $\bar{T}^{\mu A}:=\overline{T_{B}{ }^{\mu}} \Xi^{B A}$ we define a dual bi-supertwistor as

$$
\bar{X}^{A B}:=\varepsilon_{\mu \nu} \bar{T}^{\mu A} \bar{T}^{\nu B}=-(-1)^{\varepsilon_{A} \varepsilon_{B}} X^{B A} .
$$

The supermatrices $X=\left(X_{A B}\right)$ and $\bar{X}=\left(\bar{X}^{A B}\right)$ are related to each other as

$$
\bar{X}^{A B}=-(-1)^{\varepsilon_{C}} \Xi^{A C}\left(X^{\dagger}\right)_{C D} \Xi^{D B}, \quad\left(X^{\dagger}\right)_{A B}:=\overline{X_{B A}} .
$$

In the case that $T_{A}{ }^{\mu}$ generate a null two-plane, the associated bi-supertwistor $X_{A B}$ has the following properties:

$$
\begin{aligned}
X_{[A B} X_{C D\}} & =0, \\
\Upsilon^{B A} X_{A B} & =0, \\
\bar{X}^{A B} X_{B C} & =0 .
\end{aligned}
$$

In terms of $X_{A B}$, the reality conditions (4.19) take the form:

$$
\left(\Upsilon^{-1}\right)_{A C}\left(\Upsilon^{-1}\right)_{B D} \bar{X}^{C D} \propto X_{A B}
$$

The above consideration naturally leads to a new realization of the supersphere $S^{3 \mid 4 n}$. In the space of graded antisymmetric supermatrices $X_{A B}=-(-1)^{\varepsilon_{A} \varepsilon_{B}} X_{B A}$, we consider a surface $\mathfrak{L}$ spanned by those supermatrices which (i) obey the algebraic constraints (4.24); (ii) satisfy the reality condition (4.25); and (iii) have the property that the body of the bosonic block $X_{\hat{\alpha} \hat{\beta}}$ defined by

$$
X_{A B}=\left(\begin{array}{c|c}
X_{i j} & X_{i \hat{\beta}} \\
\hline X_{\hat{\alpha} j} & X_{\hat{\alpha} \hat{\beta}}
\end{array}\right)
$$

is a non-zero antisymmetric $4 \times 4$ matrix. It may be shown ${ }^{10}$ that the quotient space of $\mathfrak{L}$ with respect to $(4.21)$ is equivalent to $S^{3 \mid 4 n}$.

\footnotetext{
${ }^{10}$ The proof is analogous to the one given in [11] in the Lorentzian case.
} 


\subsection{Atlas on the supersphere}

Now we introduce an atlas on $S^{3 \mid 4 n}$ as a natural generalization of the bosonic construction described in subsection 3.4. A bi-product of our consideration in this subsection will be a formalism to describe the superconformal transformations in flat Euclidean superspace $\mathbb{E}^{3 \mid 4 n}$.

It is advantageous to introduce a new parametrization of the superconformal group $\operatorname{OSp}(2 n \mid 2,2)$ obtained by applying a similarity transformation associated with the $(2 n \mid 4) \times$ $(2 n \mid 4)$ supermatrix (2.16). The similarity transformation is defined as

$$
\begin{aligned}
g & \rightarrow \boldsymbol{g}=\boldsymbol{\Sigma} g \boldsymbol{\Sigma}^{-1}, \quad g \in \operatorname{OSp}(2 n \mid 2,2) \\
T & \rightarrow \boldsymbol{T}=\boldsymbol{\Sigma} T
\end{aligned}
$$

for any pure supertwistor $T$.

The null two-plane (4.17) turns into

$$
\mathcal{P}=\left(\begin{array}{c}
\Theta \\
\boldsymbol{h} \\
\mathbb{1}_{2}
\end{array}\right) \rightarrow \mathcal{P}=\frac{1}{\sqrt{2}}\left(\begin{array}{c}
\frac{\sqrt{2} \Theta}{\boldsymbol{h}-\mathbb{1}_{2}} \\
\boldsymbol{h}+\mathbb{1}_{2}
\end{array}\right)
$$

A natural atlas on $S^{3 \mid 4 n}$ consists of two charts, $S^{3 \mid 4 n}=U_{\mathrm{N}} \bigcup U_{\mathrm{S}}$, where the open sets $U_{\mathrm{N}}$ and $U_{\mathrm{S}}$ are defined by the conditions $\operatorname{det}\left(\boldsymbol{h}+\mathbb{1}_{2}\right) \neq 0$ and $\operatorname{det}\left(\boldsymbol{h}-\mathbb{1}_{2}\right) \neq 0$, respectively. In the north chart, the above two-plane is equivalently described by

$$
\mathcal{P} \sim\left(\begin{array}{c}
\boldsymbol{\theta}_{\mathrm{N}} \\
\mathrm{i} \boldsymbol{x}_{\mathrm{N}} \\
\mathbb{1}_{2}
\end{array}\right), \quad \mathrm{i} \boldsymbol{x}_{\mathrm{N}}:=\frac{\boldsymbol{h}-\mathbb{1}_{2}}{\boldsymbol{h}+\mathbb{1}_{2}}, \quad \boldsymbol{\theta}_{\mathrm{N}}:=\sqrt{2} \Theta\left(\boldsymbol{h}+\mathbb{1}_{2}\right)^{-1}
$$

In the south chart, the same two-plane is parametrized by

$$
\mathcal{P} \sim\left(\begin{array}{c}
\boldsymbol{\theta}_{\mathrm{S}} \\
\mathbb{1}_{2} \\
\mathrm{i} \boldsymbol{x}_{\mathrm{S}}
\end{array}\right), \quad \mathrm{i} \boldsymbol{x}_{\mathrm{S}}:=\frac{\boldsymbol{h}+\mathbb{1}_{2}}{\boldsymbol{h}-\mathbb{1}_{2}}, \quad \boldsymbol{\theta}_{\mathrm{S}}:=\sqrt{2} \Theta\left(\boldsymbol{h}-\mathbb{1}_{2}\right)^{-1}
$$

In the overlap of the two charts, $U_{\mathrm{N}} \bigcap U_{\mathrm{S}}$, we obtain the transition functions

$$
\boldsymbol{x}_{\mathrm{S}}=-\boldsymbol{x}_{\mathrm{N}}^{-1}, \quad \boldsymbol{\theta}_{\mathrm{S}}=-\mathrm{i} \boldsymbol{\theta}_{\mathrm{N}} \boldsymbol{x}_{\mathrm{N}}^{-1} .
$$

The point $\infty_{\mathrm{S}} \in U_{\mathrm{N}}$ labeled by $\boldsymbol{x}_{\mathrm{N}}=0$ and $\boldsymbol{\theta}_{\mathrm{N}}=0$ is infinitely separated from the point of view of $U_{\mathrm{S}}$. Similarly, the point $\infty_{\mathrm{N}} \in U_{\mathrm{S}}$ parametrized by $\boldsymbol{x}_{\mathrm{S}}=0$ and $\boldsymbol{\theta}_{\mathrm{S}}=0$ is infinitely separated for any observer in $U_{\mathrm{N}}$.

In what follows, we will mostly work in the north chart and omit the subscript ' $N$ ' if no confusion may occur. In the north chart, the null conditions (4.18) become

$$
\begin{aligned}
\boldsymbol{\theta}^{\dagger} \Omega \boldsymbol{\theta}+\mathrm{i}\left(\boldsymbol{x}-\boldsymbol{x}^{\dagger}\right) & =0 ; \\
-\boldsymbol{\theta}^{\mathrm{T}} \boldsymbol{\theta}+\mathrm{i}\left(\sigma_{2} \boldsymbol{x}+\boldsymbol{x}^{\mathrm{T}} \sigma_{2}\right) & =0 .
\end{aligned}
$$


The reality conditions (4.19) turn into

$$
\begin{aligned}
\overline{\boldsymbol{\theta}} & =-\Omega \boldsymbol{\theta} \sigma_{2} \\
\overline{\boldsymbol{x}} & =-\sigma_{2} \boldsymbol{x} \sigma_{2} .
\end{aligned}
$$

It follows from eqs. (4.32) and (4.33) that

$$
\boldsymbol{x}=x+\frac{\mathrm{i}}{2} \boldsymbol{\theta}^{\dagger} \Omega \boldsymbol{\theta}=x+\frac{\mathrm{i}}{4} \operatorname{tr}\left(\boldsymbol{\theta}^{\dagger} \Omega \boldsymbol{\theta}\right) \mathbb{1}_{2}, \quad x=\vec{x} \cdot \vec{\sigma}, \quad \vec{x} \in \mathbb{R}^{3} .
$$

Thus the chart $U_{\mathrm{N}}$ may be identified with a superspace $\mathbb{R}^{3 \mid 4 n}$.

\subsection{Superconformal transformations}

In the matrix realization (4.27), any element $\mathcal{L}$ of the superconformal algebra $\mathfrak{o s p}(2 n \mid 2,2)$ obeys the equations

$$
\mathcal{L}^{\dagger} \boldsymbol{\Xi}+\boldsymbol{\Xi} \mathcal{L}=0, \quad \mathcal{L}^{\mathrm{sT}} \Upsilon \mathbf{\Upsilon}+\boldsymbol{\Upsilon} \mathcal{L}=0 .
$$

The general solution of these equations in the chosen parametrization is

$$
\mathcal{L}=\left(\begin{array}{c|cc}
u & \boldsymbol{\eta} & \boldsymbol{\epsilon} \\
\hline-\boldsymbol{\epsilon}^{\dagger} \Omega & \frac{1}{2} \lambda \mathbb{1}_{2}+\mathrm{i} \vec{a} \cdot \vec{\sigma} & \mathrm{i} \vec{b} \cdot \vec{\sigma} \\
-\boldsymbol{\eta}^{\dagger} \Omega & \mathrm{i} \vec{c} \cdot \vec{\sigma} & -\frac{1}{2} \lambda \mathbb{1}_{2}+\mathrm{i} \vec{a} \cdot \vec{\sigma}
\end{array}\right), \quad u \in \mathfrak{s o}^{*}(2 n) .
$$

Here the bosonic parameters $\lambda$ and $\vec{a}, \vec{b}, \vec{c}$ are real, while the fermionic parameters obey the pseudo-Majorana condition

$$
\overline{\boldsymbol{\epsilon}}=-\Omega \boldsymbol{\epsilon} \sigma_{2}, \quad \overline{\boldsymbol{\eta}}=-\Omega \boldsymbol{\eta} \sigma_{2} .
$$

In what follows, we will use the condensed notation

$$
\mathfrak{a}:=\vec{a} \cdot \vec{\sigma}, \quad \mathfrak{b}:=\vec{b} \cdot \vec{\sigma}, \quad \mathfrak{c}:=\vec{c} \cdot \vec{\sigma}, \quad \vec{a}, \vec{b}, \vec{c} \in \mathbb{R}^{3}
$$

for the parameters in (4.36).

Similar to the bosonic case, eq. (3.32), the superconformal group acts on $S^{3 \mid 4 n}$ by fractional linear transformations. In the infinitesimal case, the superconformal transformation of $S^{3 \mid 4 n}$ associated with $\mathcal{L}$, eq. (4.36), is

$$
\begin{aligned}
\delta \boldsymbol{x} & =\mathfrak{b}+\lambda \boldsymbol{x}+\mathrm{i}[\mathfrak{a}, \boldsymbol{x}]+\mathrm{i} \boldsymbol{\epsilon}^{\dagger} \Omega \boldsymbol{\theta}+\boldsymbol{x} \mathfrak{c} \boldsymbol{x}+\boldsymbol{x} \boldsymbol{\eta}^{\dagger} \Omega \boldsymbol{\theta}, \\
\delta \boldsymbol{\theta} & =\boldsymbol{\epsilon}+\frac{1}{2} \lambda \boldsymbol{\theta}+u \boldsymbol{\theta}-\mathrm{i} \boldsymbol{\theta} \mathfrak{a}+\mathrm{i} \boldsymbol{\eta} \boldsymbol{x}+\boldsymbol{\theta} \boldsymbol{\eta}^{\dagger} \Omega \boldsymbol{\theta}+\boldsymbol{\theta} \mathfrak{c} \boldsymbol{x} .
\end{aligned}
$$

Using these expressions, we can read off the superconformal transformation of the bosonic coordinates $x^{a}$ by representing $x=\vec{x} \cdot \vec{\sigma}=\left(x_{\alpha}{ }^{\beta}\right)$ in the form $x=\boldsymbol{x}-\frac{\mathrm{i}}{2} \boldsymbol{\theta}^{\dagger} \Omega \boldsymbol{\theta}$.

The isotropy group of the point $\infty_{\mathrm{N}} \in S^{3 \mid 4 n}$ is generated by those supermatrices (4.36) for which $\boldsymbol{\eta}=0$ and $\vec{c}=0$. The most general element of the isotropy group of $\infty_{\mathrm{N}}$ is the product of a block-diagonal supermatrix

$$
\left(\begin{array}{c|cc}
\mathbb{1}_{2 n} & 0 & 0 \\
\hline 0 & \mathrm{e}^{\frac{1}{2} \lambda} \mathbb{1}_{2} & 0 \\
0 & 0 & \mathrm{e}^{-\frac{1}{2} \lambda_{1}} \mathbb{1}_{2}
\end{array}\right)\left(\begin{array}{c|cc}
\mathfrak{U} & 0 & 0 \\
\hline 0 & \mathbb{1}_{2} & 0 \\
0 & 0 & \mathbb{1}_{2}
\end{array}\right)\left(\begin{array}{c|cc}
\mathbb{1}_{2 n} & 0 & 0 \\
\hline 0 & \mathfrak{R} & 0 \\
0 & 0 & \mathfrak{R}
\end{array}\right)
$$


with a super-translation

$$
g(\mathfrak{b}, \boldsymbol{\epsilon})=\left(\begin{array}{c|cc}
\mathbb{1}_{2 n} & 0 & \boldsymbol{\epsilon} \\
\hline-\boldsymbol{\epsilon}^{\dagger} \Omega & \mathbb{1}_{2} & \mathrm{i} \boldsymbol{b} \\
0 & 0 & \mathbb{1}_{2}
\end{array}\right) .
$$

The parameters $\lambda$ and $\mathfrak{R}$ in (4.40) are the same as in (3.33), and the matrix $\mathfrak{U}$ is a group element of SO* $(2 n)$, see (2.14). The fermionic parameter $\boldsymbol{\epsilon}$ in (4.41) obeys the pseudoMajorana condition (4.37), and the bosonic $2 \times 2$ matrix $\boldsymbol{b}$ has the form

$$
\boldsymbol{b}=\mathfrak{b}+\frac{\mathrm{i}}{2} \boldsymbol{\epsilon}^{\dagger} \Omega \boldsymbol{\epsilon}=\mathfrak{b}+\frac{\mathrm{i}}{4} \operatorname{tr}\left(\boldsymbol{\epsilon}^{\dagger} \Omega \boldsymbol{\epsilon}\right) \mathbb{1}_{2},
$$

with $\mathfrak{b}$ being as in (4.38).

All transformations (4.40) also belong to the isotropy group of the point $\infty_{\mathrm{S}} \in S^{3 \mid 4 n}$, which is the origin of the chart $U_{\mathrm{N}}$. In addition, this group includes all special conformal super-translations of the form

$$
\boldsymbol{g}(\mathfrak{c}, \boldsymbol{\eta})=\left(\begin{array}{c|cc}
\mathbb{1}_{2 n} & \boldsymbol{\eta} & 0 \\
\hline 0 & \mathbb{1}_{2} & 0 \\
-\boldsymbol{\eta}^{\dagger} \Omega & \mathrm{i} \boldsymbol{c} & \mathbb{1}_{2}
\end{array}\right) .
$$

Here the fermionic parameter $\boldsymbol{\eta}$ obeys the pseudo-Majorana condition (4.37), and the bosonic $2 \times 2$ matrix $c$ has the form

$$
\boldsymbol{c}=\mathfrak{c}+\frac{\mathrm{i}}{2} \boldsymbol{\eta}^{\dagger} \Omega \boldsymbol{\eta}=\mathfrak{c}+\frac{\mathrm{i}}{4} \operatorname{tr}\left(\boldsymbol{\eta}^{\dagger} \Omega \boldsymbol{\eta}\right) \mathbb{1}_{2},
$$

where $\mathfrak{c}$ is defined by (4.38). The supermatrices (4.40), (4.41) and (4.43) generate the superconformal group $\operatorname{OSp}(2 n \mid 2,2)$. This statement is a version of the Harish-Chandra decomposition, see, e.g., [30].

The supermatrices (4.40) with $\lambda=0$ and (4.41) generate the isometry supergroup of a flat Euclidean superspace $\mathbb{E}^{3 \mid 4 n}$. As a supermanifold, this superspace may be identified with the north chart $U_{\mathrm{N}}$ of $S^{3 \mid 4 n}$. The action of the group elements (4.40) with $\lambda=0$ and (4.41) on $\mathbb{E}^{3 \mid 4 n}$ is induced by their action on $S^{3 \mid 2 n}$. In particular, the super-translation (4.41) acts on $S^{3 \mid 4 n}$ by the rule $\mathcal{P} \rightarrow \mathcal{P}^{\prime}=\boldsymbol{g}(\mathfrak{b}, \boldsymbol{\epsilon}) \mathcal{P}$, with the two-plane $\mathcal{P}$ given by (4.29). The explicit form of this transformation is

$$
x^{\prime}=x+\frac{\mathrm{i}}{2} \boldsymbol{\epsilon}^{\dagger} \Omega \boldsymbol{\theta}-\frac{\mathrm{i}}{2} \boldsymbol{\theta}^{\dagger} \Omega \boldsymbol{\epsilon}, \quad \boldsymbol{\theta}^{\prime}=\boldsymbol{\theta}+\boldsymbol{\epsilon},
$$

where we have used the transformation law $\boldsymbol{x}^{\prime}=\boldsymbol{x}+\mathrm{i} \boldsymbol{\epsilon}^{\dagger} \Omega \boldsymbol{\theta}+\frac{\mathrm{i}}{2} \boldsymbol{\epsilon}^{\dagger} \Omega \boldsymbol{\epsilon}$.

Let us consider the one-form ${ }^{11}$

$$
e=\mathrm{d} x+\frac{\mathrm{i}}{2} \mathrm{~d} \boldsymbol{\theta}^{\dagger} \Omega \boldsymbol{\theta}-\frac{\mathrm{i}}{2} \boldsymbol{\theta}^{\dagger} \Omega \mathrm{d} \boldsymbol{\theta}, \quad e_{\alpha}^{\beta}=\left(\sigma^{a}\right)_{\alpha}{ }^{\beta} e_{a} .
$$

The SO* $(2 n)$ transformations (4.40) and the super-translations (4.41) leave this one-form invariant. Under the $\mathfrak{R}$-transformations (4.40), the one-form changes as $e^{\prime}=\mathfrak{R} e \mathfrak{R}^{-1}$. As a result, all transformations (4.40) with $\lambda=0$ and (4.41) leave invariant the metric

$$
\mathrm{d} s_{\text {flat }}^{2}:=e^{a} e_{a},
$$

and therefore these transformations are indeed isometries of $\mathbb{E}^{3 \mid 4 n}$.

\footnotetext{
${ }^{11}$ This one-form is a Euclidean 3D version of the Volkov-Akulov supersymmetric one-form [27-29].
} 


\subsection{Superconformal metric}

Let us introduce a matrix two-point function on $S^{3 \mid 4 n}$

$$
\mathcal{E}(1,2):=\mathcal{P}_{1}^{\dagger} \Xi \mathcal{P}_{2}=\Theta_{1}^{\dagger} \Omega_{2} \Theta_{2}+\boldsymbol{h}_{1}^{\dagger} \boldsymbol{h}_{2}-\mathbb{1}_{2},
$$

where $\mathcal{P}$ is defined by (4.17). Given a group element $g \in \operatorname{OSp}(2 \mid 2,2)$, it acts on $S^{3 \mid 4 n}$ by the rule

$$
g\left(\begin{array}{c}
\Theta \\
\boldsymbol{h} \\
\mathbb{1}_{2}
\end{array}\right)=\left(\begin{array}{c}
\frac{\Theta^{\prime}}{\boldsymbol{h}^{\prime}} \\
\mathbb{1}_{2}
\end{array}\right) \varphi(g, \Theta, \boldsymbol{h}), \quad \varphi(g, \Theta, \boldsymbol{h}) \in \mathrm{GL}(2, \mathbb{C}) .
$$

This means that $\mathcal{E}(1,2)$ transforms homogeneously,

$$
\mathcal{E}\left(1^{\prime}, 2^{\prime}\right)=\left(\varphi^{\dagger}(g, 1)\right)^{-1} \mathcal{E}(1,2)(\varphi(g, 2))^{-1} .
$$

Associated with $\mathcal{E}(1,2)$ is the two-point function $\Delta(1,2):=\operatorname{det} \mathcal{E}(1,2)$ with the superconformal transformation law

$$
\Delta\left(1^{\prime}, 2^{\prime}\right)=\Delta(1,2)(\overline{\operatorname{det} \varphi(g, 1)} \operatorname{det} \varphi(g, 2))^{-1} .
$$

Let us choose $\Theta_{1}=\Theta, \boldsymbol{h}_{1}=\boldsymbol{h}$ and $\Theta_{2}=\Theta+\mathrm{d} \Theta, \boldsymbol{h}_{2}=\boldsymbol{h}+\mathrm{d} \boldsymbol{h}$ in the definition (4.48). This gives the one-form

$$
\mathcal{E}=\Theta^{\dagger} \Omega \mathrm{d} \Theta+\boldsymbol{h}^{\dagger} \mathrm{d} \boldsymbol{h}=-\sigma_{2} \Theta^{\mathrm{T}} \mathrm{d} \Theta+\sigma_{2} \boldsymbol{h}^{\mathrm{T}} \sigma_{2} d \boldsymbol{h}, \quad \mathcal{E}^{\dagger}=-\mathcal{E}
$$

with the superconformal transformation

$$
\mathcal{E}^{\prime}=\left(\varphi^{\dagger}\right)^{-1} \mathcal{E} \varphi^{-1}, \quad \varphi \equiv \varphi(g, \Theta, \boldsymbol{h}) .
$$

Introducing a super-interval

$$
\mathrm{d} s^{2}:=\frac{1}{4} \operatorname{det} \mathcal{E},
$$

it follows that it only scales under the superconformal transformations,

$$
\mathrm{d} s^{2} \rightarrow \mathrm{d} s^{2}|\operatorname{det} \varphi|^{-2} .
$$

By construction, the super-interval is invariant under the subgroup $\operatorname{OSp}(2 n \mid 2) \times \operatorname{SU}(2) \subset$ $\operatorname{OSp}(2 n \mid 2,2)$ which consists of those group elements which leave invariant the non-null two-plane

$$
\left(\begin{array}{c}
0 \\
0 \\
\mathbb{1}_{2}
\end{array}\right)
$$

In the north chart, a direct calculation of $\mathcal{E}$ gives the following expression:

$$
\mathcal{E}=2 \mathrm{i}\left(\mathbb{1}_{2}+\mathrm{i} \boldsymbol{x}^{\dagger}\right)^{-1} e\left(\mathbb{1}_{2}-\mathrm{i} \boldsymbol{x}\right)^{-1},
$$


where $e=\left(e_{\alpha}{ }^{\beta}\right)$ is the rigid supersymmetric one-form (4.46). ${ }^{12}$ As a result, the superinterval is

$$
\mathrm{d} s^{2}=\frac{e^{a} e_{a}}{\left|\operatorname{det}\left(\mathbb{1}_{2}-\mathrm{i} \boldsymbol{x}\right)\right|^{2}} .
$$

Switching off the Grassmann coordinates in (4.58) gives a conformally covariant and SO(4) invariant metric on $S^{3}$. The supermetric (4.58) is a smooth tensor field over $S^{3 \mid 4 n}$.

\section{$5 \mathcal{N}=2$ supersphere}

In this section we study the $n=1$ case. Its special feature is that the $R$-symmetry subgroup of $\operatorname{OSp}(2 \mid 2,2)$ is compact, $\mathrm{SO}^{*}(2) \cong \mathrm{U}(1)$. For all other values of $n>1$, the $R$-symmetry subgroup $\mathrm{SO}^{*}(2 n)$ of the superconformal group $\operatorname{OSp}(2 n \mid 2,2)$ is non-compact. Since for $n=1$ the most general expression for $\Omega$ is $\pm \sigma_{2}$, without loss of generality we choose $\Omega=\sigma_{2}$.

It is useful to introduce new Grassmann coordinates, $\boldsymbol{\theta}_{i}{ }^{\alpha} \rightarrow \hat{\boldsymbol{\theta}}_{i}{ }^{\alpha}$, that have definite $\mathrm{U}(1)_{R}$ charges. They are defined as

$$
\hat{\boldsymbol{\theta}}=\left(\hat{\boldsymbol{\theta}}_{i}{ }^{\beta}\right) \equiv\left(\begin{array}{c}
\theta^{\beta} \\
\bar{\theta}^{\beta}
\end{array}\right):=T \boldsymbol{\theta}, \quad T=\frac{1}{\sqrt{2}}\left(\begin{array}{ll}
1 & \mathrm{i} \\
\mathrm{i} & 1
\end{array}\right) .
$$

In this coordinate system, the super-metrics (2.18) become

$$
\hat{\boldsymbol{\Xi}}=\left(\begin{array}{c|cc}
\hat{\Omega} & 0 & 0 \\
\hline 0 & 0 & \mathbb{1}_{2} \\
0 & \mathbb{1}_{2} & 0
\end{array}\right), \quad \hat{\boldsymbol{\Upsilon}}=\left(\begin{array}{c|cc}
-\mathrm{i} \tau & 0 & 0 \\
\hline 0 & 0 & \sigma_{2} \\
0 & \sigma_{2} & 0
\end{array}\right),
$$

where $\hat{\Omega}:=T \Omega T^{-1}=-\sigma_{3}$ and $\tau=\sigma_{1}$ with $\sigma_{1}$ and $\sigma_{3}$ being the first and third Pauli matrices carrying $S O(2)$ indices. It is important to point out that $\hat{\Omega}$ is not antisymmetric, unlike $\Omega$. In the coordinate system introduced, the null conditions (4.32) take the form

$$
\begin{aligned}
\hat{\boldsymbol{\theta}}^{\dagger} \hat{\Omega} \hat{\boldsymbol{\theta}}+\mathrm{i}\left(\boldsymbol{x}-\boldsymbol{x}^{\dagger}\right) & =0 ; \\
\hat{\boldsymbol{\theta}}^{\mathrm{T}} \tau \hat{\boldsymbol{\theta}}+\sigma_{2} \boldsymbol{x}+\boldsymbol{x}^{\mathrm{T}} \sigma_{2} & =0 .
\end{aligned}
$$

The reality condition $(4.33 \mathrm{a})$ now reads

$$
\overline{\theta^{\alpha}}=\varepsilon_{\alpha \beta} \bar{\theta}^{\beta} \equiv \bar{\theta}_{\alpha}, \quad \overline{\bar{\theta}^{\alpha}}=-\varepsilon_{\alpha \beta} \theta^{\beta} \equiv-\theta_{\alpha} .
$$

To raise and lower two-component spinor indices, we use antisymmetric matrices $\varepsilon_{\alpha \beta}=$ $-\varepsilon_{\beta \alpha}$ and $\varepsilon^{\alpha \beta}=-\varepsilon^{\beta \alpha}$ normalized by $\varepsilon^{12}=-\varepsilon_{12}=1$. The spinor indices are lowered and raised according to

$$
\Psi^{\alpha} \rightarrow \Psi_{\alpha}=\varepsilon_{\alpha \beta} \Psi^{\beta}, \quad \Psi_{\alpha} \rightarrow \Psi^{\alpha}=\varepsilon^{\alpha \beta} \Psi_{\beta} .
$$

Eq. (4.34) becomes

$$
\boldsymbol{x}=x+\frac{\mathrm{i}}{2} \theta^{\gamma} \bar{\theta}_{\gamma} \mathbb{1}_{2}, \quad x=\vec{x} \cdot \vec{\sigma}, \quad \vec{x} \in \mathbb{R}^{3} .
$$

\footnotetext{
${ }^{12}$ This parametrization of the bosonic Cartan superform on $S^{3 \mid 4 n}$ is similar to a so-called GL-flat parametrization of the Cartan forms of the $\operatorname{OSp}(1 \mid 2 n, \mathbb{R})$ supergroup manifolds found in [31]. More generally, the expression (4.57) is a natural extension of those for the Cartan forms on Hermitian symmetric spaces [32].
} 


\subsection{Superconformal transformations}

Here we specify the main results of subsection 4.5 to the $n=1$ case using the Grassmann coordinate basis introduced above. The relations given in this subsection are preparatory for our subsequent analysis in the remainder of the section.

In the basis (5.1), the element (4.36) of the superconformal algebra osp $(2 \mid 2,2)$ takes the form:

$$
\hat{\mathcal{L}}=\left(\begin{array}{ccc}
-\mathrm{i} \varphi \hat{\Omega} & \hat{\boldsymbol{\eta}} & \hat{\boldsymbol{\epsilon}} \\
\hline-\hat{\boldsymbol{\epsilon}}^{\dagger} \hat{\Omega} & \frac{1}{2} \lambda \mathbb{1}_{2}+\mathrm{i} \mathfrak{a} & \mathrm{i} \mathfrak{b} \\
-\hat{\boldsymbol{\eta}}^{\dagger} \hat{\Omega} & \mathrm{ic} & -\frac{1}{2} \lambda \mathbb{1}_{2}+\mathrm{i} \mathfrak{a}
\end{array}\right), \quad \varphi \in \mathbb{R} .
$$

Here the bosonic parameters $\lambda$ and $\mathfrak{a}=\vec{a} \cdot \vec{\sigma}, \mathfrak{b}=\vec{b} \cdot \vec{\sigma}, \mathfrak{c}=\vec{c} \cdot \vec{\sigma}$ are the same as in (4.36). The fermionic $2 \times 2$ matrix $\hat{\boldsymbol{\epsilon}}$ has the structure

$$
\hat{\boldsymbol{\epsilon}}=\left(\hat{\boldsymbol{\epsilon}}_{i}{ }^{\beta}\right) \equiv\left(\begin{array}{c}
\epsilon^{\beta} \\
\bar{\epsilon}^{\beta}
\end{array}\right), \quad \overline{\epsilon^{\alpha}}=\bar{\epsilon}_{\alpha}=\varepsilon_{\alpha \beta} \bar{\epsilon}^{\beta}, \quad \overline{\bar{\epsilon}^{\alpha}}=-\epsilon_{\alpha},
$$

and similar for $\hat{\boldsymbol{\eta}}$. The parameter $\varphi$ describes a $\mathrm{U}(1)_{R}$ transformation. The $\mathrm{U}(1)_{R}$ charge of $\theta^{\alpha}$ is +1 . As follows from (4.39), the most general infinitesimal superconformal transformation in the north chart of $S^{3 \mid 4}$ is

$$
\begin{aligned}
\delta \boldsymbol{x} & =\mathfrak{b}+\lambda \boldsymbol{x}+\mathrm{i}[\mathfrak{a}, \boldsymbol{x}]+\mathrm{i} \hat{\boldsymbol{\epsilon}}^{\dagger} \hat{\Omega} \hat{\boldsymbol{\theta}}+\boldsymbol{x} \mathfrak{c} \boldsymbol{x}+\boldsymbol{x} \hat{\boldsymbol{\eta}}^{\dagger} \hat{\Omega} \hat{\boldsymbol{\theta}} \\
\delta \hat{\boldsymbol{\theta}} & =\hat{\boldsymbol{\epsilon}}+\frac{1}{2} \lambda \hat{\boldsymbol{\theta}}-\mathrm{i} \varphi \hat{\Omega} \hat{\boldsymbol{\theta}}-\mathrm{i} \hat{\boldsymbol{\theta}} \mathfrak{a}+\mathrm{i} \hat{\boldsymbol{\eta}} \boldsymbol{x}+\hat{\boldsymbol{\theta}} \hat{\boldsymbol{\eta}}^{\dagger} \hat{\Omega} \hat{\boldsymbol{\theta}}+\hat{\boldsymbol{\theta}} \mathfrak{c} \boldsymbol{x} .
\end{aligned}
$$

The super-translation (4.41) takes the form

$$
\hat{\boldsymbol{g}}(\mathfrak{b}, \hat{\boldsymbol{\epsilon}})=\left(\begin{array}{c|cc}
\mathbb{1}_{2} & 0 & \hat{\boldsymbol{\epsilon}} \\
\hline-\hat{\boldsymbol{\epsilon}}^{\dagger} \hat{\Omega} & \mathbb{1}_{2} & \mathrm{i} \boldsymbol{b} \\
0 & 0 & \mathbb{1}_{2}
\end{array}\right), \quad \boldsymbol{b}=\mathfrak{b}+\frac{\mathrm{i}}{2} \epsilon^{\gamma} \bar{\epsilon}_{\gamma} \mathbb{1}_{2} .
$$

In the north chart of $S^{3 \mid 4}$, this group element acts as follows

$$
\hat{\boldsymbol{g}}(\mathfrak{b}, \hat{\boldsymbol{\epsilon}})\left(\begin{array}{c}
\hat{\boldsymbol{\theta}} \\
\mathrm{i} \boldsymbol{x} \\
\mathbb{1}_{2}
\end{array}\right)=\left(\begin{array}{c}
\frac{\hat{\boldsymbol{\theta}}^{\prime}}{\mathrm{i} \boldsymbol{x}^{\prime}} \\
\mathbb{1}_{2}
\end{array}\right)
$$

where

$$
\boldsymbol{x}^{\prime}=\boldsymbol{x}+\boldsymbol{b}+\mathrm{i} \hat{\boldsymbol{\epsilon}}^{\dagger} \hat{\Omega} \hat{\boldsymbol{\theta}}, \quad \hat{\boldsymbol{\theta}}^{\prime}=\hat{\boldsymbol{\theta}}+\hat{\boldsymbol{\epsilon}}
$$

In terms of the coordinates $x_{\alpha}{ }^{\beta}$ and $\theta_{\alpha}$, this transformation law reads ${ }^{13}$

$$
x_{\alpha}^{\prime \beta}=x_{\alpha}{ }^{\beta}+b_{\alpha}{ }^{\beta}-\mathrm{i} \epsilon_{(\alpha} \bar{\theta}^{\beta)}-\mathrm{i} \bar{\epsilon}_{(\alpha} \theta^{\beta)}, \quad \theta_{\alpha}^{\prime}=\theta_{\alpha}+\epsilon_{\alpha} .
$$

The supersymmetric Cartan form (4.46) takes the form

$$
e_{\alpha}{ }^{\beta}=\mathrm{d} x_{\alpha}{ }^{\beta}+\mathrm{i} \bar{\theta}_{(\alpha} \mathrm{d} \theta^{\beta)}+\mathrm{i} \theta_{(\alpha} \mathrm{d} \bar{\theta}^{\beta)}, \quad e_{\alpha}^{\beta}=\left(\sigma^{a}\right)_{\alpha}{ }^{\beta} e_{a} .
$$

\footnotetext{
${ }^{13}$ The symmetrization of two spinor indices in (5.13) includes a factor of $1 / 2$.
} 


\subsection{Chiral subspace}

Let us now introduce a complex three-vector variable ${ }^{14} y^{a}$ defined by

$$
y=\boldsymbol{x}+\mathrm{i} \tilde{\theta} \bar{\theta}=\vec{y} \cdot \vec{\sigma} \quad \Longleftrightarrow \quad y_{\alpha}{ }^{\beta}=x_{\alpha}{ }^{\beta}+\mathrm{i} \theta_{(\alpha} \bar{\theta}^{\beta)} .
$$

In accordance with (5.13), the transformation law of $y$ is

$$
y_{\alpha}^{\prime}{ }^{\beta}=y_{\alpha}{ }^{\beta}+b_{\alpha}{ }^{\beta}-2 \mathrm{i} \bar{\epsilon}_{(\alpha} \theta^{\beta)}+\mathrm{i} \bar{\epsilon}_{(\alpha} \epsilon^{\beta)} .
$$

We see that the chiral variables $y^{a}$ and $\theta^{\alpha}$ form a closed subset under the supertransformations.

It is nontrivial that the chiral variables also form a closed subset under the superconformal transformations. Indeed, the infinitesimal superconformal transformation (5.9) may be used to show that the chiral variables vary as follows:

$$
\begin{aligned}
\delta y & =\mathfrak{b}+\lambda y+\mathrm{i}[\mathfrak{a}, y]+\mathrm{i} \tilde{\theta} \epsilon-\mathrm{i} \tilde{\bar{\epsilon}} \theta+y \mathfrak{c} y-(y \tilde{\bar{\eta}}) \theta-\tilde{\theta}(\bar{\eta} y), \\
\delta \theta & =\epsilon+\frac{1}{2} \lambda \theta+\mathrm{i} \varphi \theta-\mathrm{i} \theta \mathfrak{a}+\mathrm{i} \eta y+(\theta \tilde{\bar{\eta}}) \theta+\theta \mathfrak{c} y .
\end{aligned}
$$

The above property allows us to give an alternative definition of the $3 \mathrm{D} \mathcal{N}=2$ superconformal group that is analogous to the one used in [25] in the $4 \mathrm{D} \mathcal{N}=1$ super-Poincaré case. We introduce a complex superspace $\mathbb{C}^{3 \mid 2}$ parametrized by bosonic $y$ and fermionic $\theta^{\alpha}$ variables. Embedded into $\mathbb{C}^{3 \mid 2}$ is a real superspace $\mathbb{R}^{3 \mid 4}$ with coordinates $z^{A}=\left(x^{a}, \theta^{\alpha}, \bar{\theta}_{\alpha}\right)$, with $\bar{\theta}_{\alpha}:=\overline{\theta^{\alpha}}$, which is defined by

$$
y^{a}-\bar{y}^{a}=2 \mathrm{i} \mathcal{H}^{a}, \quad \mathcal{H}^{a}:=\frac{1}{2} \theta \sigma^{a} \tilde{\bar{\theta}}=\frac{1}{2} \theta^{\alpha}\left(\sigma^{a}\right)_{\alpha}{ }^{\beta} \bar{\theta}_{\beta} .
$$

An infinitesimal holomorphic transformation on $\mathbb{C}^{3 \mid 2}$,

$$
\delta y^{a}=\xi^{a}(y, \theta), \quad \delta \theta^{\alpha}=\xi^{\alpha}(y, \theta),
$$

is said to be superconformal if it preserves the real surface (5.18); that is,

$$
\xi^{a}-\bar{\xi}^{a}=\mathrm{i}\left(\sigma^{a}\right)_{\alpha}{ }^{\beta}\left(\xi^{\alpha} \bar{\theta}_{\beta}+\theta^{\alpha} \bar{\xi}_{\beta}\right),
$$

where $\bar{\xi}_{\alpha}(\bar{y}, \bar{\theta}):=\overline{\xi^{\alpha}(y, \theta)}$. It is an instructive exercise to show that the most general solution of this equation is given by (5.17).

\subsection{Complexified supersphere}

In accordance with (5.17), the superconformal group acts by holomorphic transformations on the chiral variables $\zeta_{\mathrm{N}}=\left(y_{\mathrm{N}}{ }^{a}, \theta_{\mathrm{N}}{ }^{\alpha}\right)$ defined in the north chart $U_{\mathrm{N}}$ of $S^{3 \mid 4}$. We can also introduce chiral variables $\zeta_{\mathrm{S}}=\left(y_{\mathrm{S}}{ }^{a}, \theta_{\mathrm{S}}{ }^{\alpha}\right)$ defined in the south chart $U_{\mathrm{S}}$ of $S^{3 \mid 4}$, by extending the definition (5.15) to the south chart. It is natural to wonder whether the

\footnotetext{
${ }^{14}$ In the remainder of this section, we often make use of row vectors $\theta=\left(\theta^{\alpha}\right)$ and $\bar{\theta}=\left(\bar{\theta}^{\alpha}\right)$ and column vectors $\tilde{\theta}=\left(\theta_{\alpha}\right)$ and $\tilde{\bar{\theta}}=\left(\bar{\theta}_{\alpha}\right)$.
} 
concept of chirality is just a local structure defined within a coordinate chart or if it is globally defined on $S^{3 \mid 4}$.

In the overlap of the north and south charts, $U_{\mathrm{N}} \cap U_{\mathrm{S}}$, we derive the transition functions:

$$
y_{\mathrm{S}}=-y_{\mathrm{N}}{ }^{-1}, \quad \theta_{\mathrm{S}}{ }^{\alpha}=-\mathrm{i} \theta_{\mathrm{N}}{ }^{\beta}\left(y_{\mathrm{N}}{ }^{-1}\right)_{\beta}^{\alpha} .
$$

This result shows that chirality is globally defined on $S^{3 \mid 4}$.

It is natural to introduce a complexified or chiral supersphere, $\mathbb{C} S^{3 / 2}$. It is defined to be a complex supermanifold which may be covered by two charts $W_{\mathrm{N}}$ and $W_{\mathrm{S}}$, $\mathbb{C} S^{3 \mid 2}=W_{\mathrm{N}} \cup W_{\mathrm{S}}$, such that the following properties hold: (i) each chart is diffeomorphic to complex superspace $\mathbb{C}^{3 \mid 2}$ parametrized by independent complex coordinates $\zeta=\left(y^{a}, \theta^{\alpha}\right)$; and (ii) in the overlap of the charts, $W_{\mathrm{N}} \cap W_{\mathrm{S}}$, the local coordinates are related to each other by the transition functions (5.21). The superconformal group naturally acts on $\mathbb{C} S^{3 \mid 2}$ by holomorphic transformations (5.17). The bosonic body of $\mathbb{C} S^{3 \mid 2}$ is a complexified threesphere that may be identified with the tangent bundle $T S^{3}$ of the three-sphere. ${ }^{15}$

\subsection{Superconformal inversion}

Super-inversion is a discrete transformation $I_{k}: S^{3 \mid 4} \rightarrow S^{3 \mid 4}$ defined by

$$
\theta^{\prime}=\bar{\kappa} \bar{\theta}\left(y^{\dagger}\right)^{-1}, \quad y^{\prime}=|\kappa|^{2}\left(y^{\dagger}\right)^{-1},
$$

for some non-zero parameter $\kappa$. This parameter may always be chosen to be equal to any given nonzero complex number by combing $I_{\kappa}$ with a scale and $\mathrm{U}(1)_{R}$ transformation. One may check that $\left(I_{\kappa}\right)^{2}=$ id. The super-inversion respects the defining equation of the chiral subspace,

$$
\mathrm{i}\left(y^{\prime}-y^{\prime \dagger}\right)_{\alpha}^{\beta}=2 \bar{\theta}_{(\alpha}^{\prime} \theta^{\prime \beta)} .
$$

It is an instructive exercise to show that the super-inversion is a discrete superconformal transformation in the sense that it only rescales the flat supermetric (4.47),

$$
\operatorname{tr}\left(e^{\prime}\right)^{2}=\frac{|\kappa|^{4}}{y^{2} \bar{y}^{2}} \operatorname{tr}\left(e^{2}\right)
$$

with the supersymmetric Cartan form given by (5.14). If one considers a composite transformation $I_{\kappa} \hat{\boldsymbol{g}}(\mathfrak{b}, \hat{\boldsymbol{\epsilon}}) I_{\kappa}$, with $\hat{\boldsymbol{g}}(\mathfrak{b}, \hat{\boldsymbol{\epsilon}})$ being the super-translation (5.10), the resulting transformation is a special conformal super-translation.

The above properties are analogous to those possessed by a super-inversion in the case of $4 \mathrm{D} \mathcal{N}=1$ superconformal symmetry $[25,33]$.

\section{Supercoset realizations of $\mathbb{E}^{3 \mid 4 n}$ and $S^{3 \mid 4 n}$}

In this section we give several supercoset realizations for $S^{3 \mid 4 n}$ and flat Euclidean superspace $\mathbb{E}^{3 \mid 4 n}$.

\footnotetext{
${ }^{15}$ As is known, the complexified three-sphere may be realized as a quadric in $\mathbb{C}^{4}$ defined by $\vec{Z} \cdot \vec{Z}=1$, with $\vec{Z}=\vec{X}+\mathrm{i} \vec{Y} \in \mathbb{C}^{4}$ and $\vec{X}, \vec{Y} \in \mathbb{R}^{4}$.
} 


\subsection{The super-translation subalgebra of $\mathfrak{o s p}(2 n \mid 2,2)$ and $\mathbb{E}^{3 \mid 4 n}$}

The Euclidean counterpart of the $D=3, \mathcal{N}=2 n$ super-Poincaré algebra is obtained from (2.25) by projecting the supersymmetry generators $\mathcal{Q}_{\hat{\alpha}}^{i}$ as follows

$$
\tilde{Q}^{i}=\mathcal{Q}^{i} \mathbb{P}_{04}
$$

where

$$
\mathbb{P}_{04}=\frac{1}{2}\left(\mathbb{1}+\gamma^{0} \gamma^{4}\right), \quad \mathbb{P}_{04} \mathbb{P}_{04}=\mathbb{P}_{04} .
$$

The supercharges (6.1), whose number is half the number of $\mathcal{Q}^{i}$, are transformed under the fundamental representation of the group $\mathrm{SU}(2)$ of rotations in $D=3$ labeled by the index $\alpha=1,2$. They generate a superalgebra which is obtained from (2.25) by multiplying its left and right hand sides by the projectors $\mathbb{P}_{04}$, taking into account the order of the spinor indices. Due to the anti-commutation properties of the gamma-matrices, the terms on the right hand side of (2.25) which survive this projection have the following form

$$
\left\{\tilde{Q}^{i}, \tilde{Q}^{j}\right\}=2 \delta^{i j} \sigma^{a} P_{a}, \quad\left[P_{a}, P_{b}\right]=0 .
$$

Due to the chosen realization of the gamma-matrices,

$$
\mathrm{i} \sigma^{a}=\left(\mathbb{1}-\mathbb{P}_{04}\right) \gamma^{a} \gamma^{0} \mathbb{P}_{04}=\left(\mathbb{1}-\mathbb{P}_{04}\right) \gamma^{a} \gamma^{4} \mathbb{P}_{04}
$$

can be associated with the Pauli matrices and $P_{a}=\mathrm{i}\left(L_{a 0}+L_{a 4}\right)$ is the generator of the translations in $3 d$ flat space. The projections $\left(\mathbb{1}-\mathbb{P}_{04}\right) \gamma^{a b} \mathbb{P}_{04}$ and $\left(\mathbb{1}-\mathbb{P}_{04}\right) \gamma^{04} \mathbb{P}_{04}$ vanish due to the commutation properties of the gamma-matrices.

The $\mathrm{SU}(2) \cong \mathrm{SO}(3) / \mathbb{Z}_{2}$ group, under which $\tilde{Q}^{i}$ and $P_{a}$ transform in the spinor and the vector representations, respectively, is generated by the operators $L_{a b}$, while $\mathrm{SO}^{*}(2 n)$ generated by $T^{i j}$ becomes the group of "external" $R$-symmetries of this superalgebra.

In the diagonal matrix realization ${ }^{16}$ of the projector $\mathbb{P}_{04}$,

$$
\mathbb{P}_{04}=\left(\begin{array}{ll}
0 & 0 \\
0 & \mathbb{1}
\end{array}\right)
$$

the elements of the $3 D$ super-translation group associated with the Euclidean superspace $\mathbb{E}^{3 \mid 4 n}$ are similar to (4.41),

$$
\mathbb{E}^{3 \mid 4 n}(x, \theta)=\left(\begin{array}{c|cc}
\mathbb{1}_{2 n} & 0 & \boldsymbol{\theta} \\
\hline-\boldsymbol{\theta}^{\dagger} \Omega & \mathbb{1}_{2} & \mathrm{i} \boldsymbol{x} \\
0 & 0 & \mathbb{1}_{2}
\end{array}\right),
$$

where $\boldsymbol{x}=x^{a} \sigma^{a}+\frac{\mathrm{i}}{2} \boldsymbol{\theta}^{\dagger} \Omega \boldsymbol{\theta}$ and the spinors $\boldsymbol{\theta}$ satisfy the symplectic-Majorana reality condition (4.33a) which follows from the reality condition (2.24) for the projected supercharges (6.1).

Note that the right column in (6.5) is nothing but the two-plane (4.29) which describes a point in the north chart of $S^{3 \mid 4 n}$.

\footnotetext{
${ }^{16}$ This realization is obtained from that of (2.23) by applying the similarity transformation (2.8).
} 
The superspace $\mathbb{E}^{3 \mid 4 n}$ defined in (6.5) can be regarded as a local supercoset of the superconformal group, namely

$$
\mathbb{E}^{3 \mid 4 n} \subset S^{3 \mid 4 n}=\frac{\mathrm{OSp}(2 n \mid 2,2)}{\mathrm{SO}^{*}(2 n) \times \mathrm{SU}(2) \rtimes S K},
$$

where $S K$ stands for the dilatation, conformal boosts and superconformal transformations. In other words, the stability group $\mathbb{H}$ of this coset is formed by the product of the matrices (4.40) and (4.43). We recall that $\mathbb{H}$ is the isotropy group of the point $\infty_{\mathrm{N}} \in S^{3 \mid 4 n}$ (see subsection 4.5) and the superspace $\mathbb{E}^{3 \mid 4 n}$ can be identified with

$$
\mathbb{E}^{3 \mid 4 n}=S^{3 \mid 4 n} \backslash\left\{\infty_{\mathrm{N}}\right\} .
$$

The superconformal group generated by (4.36) acts on the superspace $\mathbb{E}^{3 \mid 4 n}$ coset element (6.5) as follows

$$
\mathbb{E}^{\prime}\left(x^{\prime}, \theta^{\prime}\right)=\mathrm{e}^{\mathcal{L}} \mathbb{E}(x, \theta) \mathbb{H}^{-1}(x, \theta),
$$

where $\mathbb{H}^{-1}(x, \theta)$ is the compensating transformation from the stability group, which is required in order to bring the transformed coset element to a form similar to (6.5). One can check that the transformation (6.8) with infinitesimal parameters generates the superconformal transformations of $x$ and $\theta$ given in (4.39).

The special conformal super-translations (4.43) do not generate a well defined action on the flat superspace $\mathbb{E}^{3 \mid 4 n}$ if the body of the special conformal parameter $c^{a}$ in $\mathfrak{c}=\vec{c} \cdot \vec{\sigma}$ is non-zero. In this case some point $\left(x_{0}, \theta_{0}\right)$ from $\mathbb{E}^{3 \mid 4 n}$ is mapped to the infinitely separated point, $\infty_{\mathrm{N}}$, which means that $\mathbb{H}^{-1}\left(x_{0}, \theta_{0}\right)$ is not defined. By construction, all elements of the superconformal group generate well defined transformations on the supersphere $S^{3 \mid 4 n}$.

As we discussed in section 5.2, in the $n=1$ case in which $\mathrm{SO}^{*}(2)=\mathrm{SO}(2)$, there is a chiral subspace which transforms into itself under the super-translation and the infinitesimal superconformal transformations. In the generic $n>1$ case, there is no chiral subspace which would transform into itself under SO* $(2 n)$, since the $\mathrm{SO}^{*}(2 n)$ matrices (with $n>1$ ) do not commute with the symplectic form $\Omega$. The same conclusion also follows from the fact that the defining representation of $\mathrm{SO}^{*}(2 n)$ is irreducible for $n>1$.

\subsection{The $\operatorname{OSp}(2 n \mid 2) \times \operatorname{SU}(2)$ subalgebra of $\mathfrak{o s p}(2 n \mid 2,2)$ and $S^{3 \mid 4 n}$}

We recall that the super-interval (4.58) is invariant under a subgroup OSp $(2 n \mid 2) \times \operatorname{SU}(2)$ of the superconformal group $\operatorname{OSp}(2 n \mid 2,2)$. In the matrix realization $(2.13)$ of $\operatorname{OSp}(2 n \mid 2,2)$, this subgroup consists of those group elements which leave invariant the two-plane (4.56). The bosonic subgroup of the supergroup $\operatorname{OSp}(2 n \mid 2)$ is $\mathrm{SO}^{*}(2 n) \times \mathrm{Sp}(2)$, where $\mathrm{Sp}(2) \cong \mathrm{SU}(2)$.

To get an $\operatorname{OSp}(2 n \mid 2) \times \operatorname{SU}(2)$ sub-superalgebra of the $\operatorname{OSp}(2 n \mid 2,2)$ superconformal algebra (2.25), one may single out half of the supergenerators $\mathcal{Q}^{i}$ using the projector

$$
\mathbb{P}_{0}=\frac{1}{2}\left(\mathbb{1}+\gamma_{0}\right), \quad \mathbb{P}_{0} \mathbb{P}_{0}=\mathbb{P}_{0},
$$

as follows

$$
Q_{\hat{\alpha}}^{i}=\left(\mathcal{Q}^{i} \mathbb{P}_{0}\right)_{\hat{\alpha}}=\left(Q_{\alpha}^{i}, 0\right), \quad \alpha=1,2,
$$

where the index $\alpha$ corresponds to the $\mathrm{SU}(2) \cong \mathrm{Sp}(2)$ subgroup of $\operatorname{OSp}(2 n \mid 2)$. 
The spinors $Q_{\alpha}^{i}$ satisfy the symplectic-Majorana condition

$$
Q^{i \alpha}=-\mathrm{i} \varepsilon^{\alpha \beta} \Omega^{i j} \bar{Q}_{\beta}^{j} \equiv-\mathrm{i} \Omega^{i j} \bar{Q}^{j \alpha}=-\mathrm{i} \Omega^{i j}\left(Q_{\alpha}^{j}\right)^{*} .
$$

Multiplying both sides of $(2.25)$ by $\mathbb{P}_{0}$ and taking into account the order of the indices we get

$$
\left\{Q_{\alpha}^{i}, Q_{\beta}^{j}\right\}=\delta^{i j}\left(-\sigma_{\alpha \beta}^{a b} L_{a b}+2 \mathrm{i} \sigma_{\alpha \beta}^{a} L_{a 4}\right)+\epsilon_{\alpha \beta} T^{i j},
$$

where

$$
\sigma^{a} \equiv \mathrm{i} \mathbb{P}_{0} \gamma^{a} \gamma^{4} \mathbb{P}_{0}, \quad \sigma^{a b} \equiv-\mathbb{P}_{0} \gamma^{a b} \mathbb{P}_{0}
$$

can be associated with the Pauli matrices and $\epsilon=\mathrm{i} \sigma_{2}=\mathbb{P}_{0} C \mathbb{P}_{0}$, while $\mathbb{P}_{0} \gamma^{\hat{0} 0} \mathbb{P}_{0}=0$, since $\gamma^{a}$ and $\gamma^{4}$ anticommute with $\gamma^{0}$ inside $\mathbb{P}_{0}$.

Furthermore, using the identity $\sigma^{a b}=\mathrm{i} \varepsilon^{a b c} \sigma_{c}$, we may rewrite (6.12) as follows

$$
\left\{Q_{\alpha}^{i}, Q_{\beta}^{j}\right\}=\delta^{i j} \sigma_{\alpha \beta}^{a} M_{a}+\epsilon_{\alpha \beta} T^{i j},
$$

where

$$
M_{a}=\mathrm{i}\left(L_{a 4}-\frac{1}{2} \varepsilon_{a b c} L^{b c}\right)
$$

generate the $\mathrm{SU}(2)$ algebra

$$
\left[M_{a}, M_{b}\right]=2 \mathrm{i} \varepsilon_{a b c} M_{c} .
$$

We see that the generators $\tilde{M}_{a}=\mathrm{i}\left(\frac{1}{2} \varepsilon_{a b c} L^{b c}+L_{a 4}\right)$ of another $\mathrm{SU}(2)$ subalgebra of $\operatorname{OSp}(2 n \mid 2,2)$ do not appear in the right hand side of (6.14) and thus commute with those of the $\operatorname{OSp}(2 n \mid 2)$.

In the $n=1$ case, the superalgebra isomorphism $\mathfrak{o s p}(2 \mid 2) \cong \mathfrak{s u}(2 \mid 1)$ holds. Introducing the complex conjugate supercharges

$$
Q_{\alpha}=\frac{1}{\sqrt{2}}\left(Q_{\alpha}^{1}+\mathrm{i} Q_{\alpha}^{2}\right), \quad \bar{Q}_{\alpha}=-\frac{1}{\sqrt{2}}\left(Q_{\alpha}^{1}-\mathrm{i} Q_{\alpha}^{2}\right), \quad\left(Q^{\alpha}\right)^{*}=\bar{Q}_{\alpha},
$$

the anti-commutation relations take the form

$$
\left\{Q_{\alpha}, \bar{Q}_{\beta}\right\}=\sigma_{\alpha \beta}^{m} M_{m}+\epsilon_{\alpha \beta} R, \quad\left\{Q_{\alpha}, Q_{\beta}\right\}=0,
$$

where $R$ is the $\mathrm{U}(1) R$-symmetry generator.

In accordance with the above consideration, every element $M \in \mathfrak{o s p}(2 n \mid 2)$ is singled out from some element of the superconformal algebra, $\mathcal{M} \in \mathfrak{o s p}(2 n \mid 2,2)$, by multiplying the latter (from the left and from the right) with the projector

$$
\mathcal{P}_{0}=\left(\begin{array}{cc}
\mathbb{1}_{2 n} & 0 \\
0 & \mathbb{P}_{0}
\end{array}\right),
$$

namely

$$
M=\mathcal{P}_{0} \mathcal{M} \mathcal{P}_{0},
$$

where $\mathbb{1}_{2 n}$ is the unit matrix acting on the $\mathrm{SO}^{*}(2 n)$ indices. 
The supersphere can be identified with the coset superspace

$$
\mathbb{S}^{3 \mid 4 n}=\frac{\mathrm{OSp}(2 n \mid 2)}{\mathrm{SO}^{*}(2 n)}
$$

which is formed by the equivalence classes

$$
\mathrm{e}^{M} \sim \mathrm{e}^{M} h, \quad h \in \mathrm{SO}^{*}(2 n),
$$

where $M \in \mathfrak{o s p}(2 n \mid 2)$ is given by (6.20).

In the gamma-matrix realization (2.23) in which

$$
\mathbb{P}_{0}=\left(\begin{array}{cc}
\mathbb{1}_{2} & 0 \\
0 & 0
\end{array}\right)
$$

the algebra-valued element (6.20) associated with the $\mathbb{S}^{3 \mid 4 n}$ coset generators of the supergroup $\operatorname{OSp}(2 n \mid 2)$ is

$$
\mathbf{s}^{3 \mid 4 n}=\left(\begin{array}{ccc}
0 & \Theta & 0 \\
-\Theta^{\dagger} \Omega & \mathrm{ix} & 0 \\
0 & 0 & 0
\end{array}\right)
$$

where $\Theta$ are subject to the symplectic-Majorana condition (4.19) and $\mathbf{x}=\left(\mathbf{x}_{\alpha}{ }^{\beta}\right)$ is a traceless Hermitian matrix. We see that the rank of (6.24) reduces to $2 n+2$.

The supersphere $S^{3 \mid 4 n}$ parametrized by (6.24) can also be regarded as a supercoset of the conformal group $\operatorname{OSp}(2 n \mid 2,2)$ in its realization defined in $(2.13)$, which is different from (6.6). The relevant supercoset is

$$
\mathbb{S}^{3 \mid 4 n}=\left\{\mathrm{e}^{\mathbf{s}^{3 \mid 4 n}}\right\}=\frac{\mathrm{OSp}(2 n \mid 2,2)}{\mathrm{SO}^{*}(2 n) \times \mathrm{SU}(2) \rtimes S K},
$$

where, as in (6.6), $S K$ stands for the dilatation, conformal boosts and superconformal transformations. The stability group $\hat{\mathbb{H}}=\mathrm{SO}^{*}(2 n) \times \mathrm{SU}(2) \rtimes S K$ of this coset is formed by the product of the matrices (4.40) and (4.43) (as in (6.6) but) subject to the similarity transformation with the inverse matrix of (2.16), namely

$$
\hat{\mathbb{H}}=\Sigma^{-1} \mathbb{H} \Sigma .
$$

The superconformal transformation of the supercoset (6.25) is

$$
\mathbb{S}\left(\mathbf{x}^{\prime}, \Theta^{\prime}\right)=\left(\boldsymbol{\Sigma}^{-1} \mathrm{e}^{\mathcal{L}} \boldsymbol{\Sigma}\right) \mathbb{S}(\mathbf{x}, \Theta) \hat{\mathbb{H}}^{-1}(\mathbf{x}, \Theta)
$$

where $\mathcal{L}$ is the same as in (4.36).

The supercoset element associated with (6.24) parametrizing the points of the supersphere $S^{3 \mid 4 n}$ can be given in the form

$$
\mathbb{S}=\left(\begin{array}{cc}
\mathbf{M} & \Theta \\
-\boldsymbol{h} \Theta^{\dagger} \Omega \mathbf{M}^{-1} & \boldsymbol{h}
\end{array}\right)=\left(\begin{array}{cc}
\mathbf{M} & \Theta \\
\sigma_{2}\left(\boldsymbol{h}^{-1}\right)^{\mathrm{T}} \Theta^{\mathrm{T}} \mathbf{M} & \boldsymbol{h}
\end{array}\right)
$$


where $\boldsymbol{h}$ satisfies the constraints (4.18) and (4.19), while $\mathbf{M}$ is defined by

$$
\mathbf{M}:=\left(\mathbb{1}_{2 n}-\Theta \Theta^{\dagger} \Omega\right)^{\frac{1}{2}}=\left(\mathbb{1}_{2 n}+\Theta \sigma_{2} \Theta^{\mathrm{T}}\right)^{\frac{1}{2}}=\Omega \mathbf{M}^{\dagger} \Omega,
$$

such that $\left(\mathbf{M}^{2}\right)_{i}{ }^{j}=\delta_{i}^{j}+\mathrm{i} \Theta_{i}^{\alpha} \Theta_{\alpha}^{j}$. The right column of (6.28) involves the same matrix blocks $\Theta$ and $\boldsymbol{h}$ which constitute the null two-plane (4.17). The inverse of $\mathbb{S}$ is

$$
\mathbb{S}^{-1}=\left(\begin{array}{cc}
\mathbf{M} & -\mathbf{M}^{-1} \Theta \boldsymbol{h}^{\dagger} \\
\Theta^{\dagger} \Omega & \boldsymbol{h}^{\dagger}
\end{array}\right)=\left(\begin{array}{cc}
\mathbf{M} & -\mathbf{M} \Theta \boldsymbol{h}^{-1} \\
-\sigma_{2} \Theta^{\mathrm{T}} & \boldsymbol{h}^{\dagger}
\end{array}\right) .
$$

For completeness, here we give the most general element of $\operatorname{OSp}(2 n \mid 2)$ :

$$
g=\left(\begin{array}{c|c}
\mathbf{M} \mathfrak{U} & \Theta \\
\hline \sigma_{2}\left(\boldsymbol{h}^{-1}\right)^{\mathrm{T}} \Theta^{\mathrm{T}} \mathbf{M} \mathfrak{U} & \boldsymbol{h}
\end{array}\right), \quad \mathfrak{U} \in \mathrm{SO}^{*}(2 n) .
$$

The coset representative (6.28) is obtained from (6.31) by setting $\mathfrak{U}=\mathbb{1}_{2 n}$.

The Cartan form describing the geometry of $S^{3 \mid 4 n}$ in this realization is

$$
\begin{aligned}
\mathbf{S}^{-1} \mathrm{~d} \mathbf{S} & =\left(\begin{array}{cc}
\mathbf{M} \mathrm{d} \mathbf{M}+\mathbf{M}^{-1} \Theta \boldsymbol{h}^{\dagger} \mathrm{d}\left(\boldsymbol{h} \Theta^{\dagger} \Omega \mathbf{M}^{-1}\right) & \mathbf{M} \mathrm{d} \Theta-\mathbf{M}^{-1} \Theta \boldsymbol{h}^{\dagger} \mathrm{d} \boldsymbol{h} \\
\Theta^{\dagger} \Omega \mathrm{d} \mathbf{M}-\boldsymbol{h}^{\dagger} \mathrm{d}\left(\boldsymbol{h} \Theta^{\dagger} \Omega \mathbf{M}^{-1}\right) & \boldsymbol{h}^{\dagger} \mathrm{d} \boldsymbol{h}+\Theta^{\dagger} \Omega \mathrm{d} \Theta
\end{array}\right) \\
& =\left(\begin{array}{cc}
\mathbf{M} \mathrm{d} \mathbf{M}-\mathbf{M} \Theta \boldsymbol{h}^{-1} \mathrm{~d}\left(\sigma_{2}\left(\boldsymbol{h}^{-1}\right)^{\mathrm{T}} \Theta^{\mathrm{T}} \mathbf{M}\right) & \mathbf{M}\left(\mathrm{d} \Theta-\Theta \boldsymbol{h}^{-1} \mathrm{~d} \boldsymbol{h}\right) \\
-\sigma_{2} \Theta^{\mathrm{T}} \mathrm{d} \mathbf{M}+\boldsymbol{h}^{\dagger} \mathrm{d}\left(\sigma_{2}\left(\boldsymbol{h}^{-1}\right)^{\mathrm{T}} \Theta^{\mathrm{T}} \mathbf{M}\right) & \boldsymbol{h}^{\dagger} \mathrm{d} \boldsymbol{h}+\Theta^{\dagger} \Omega \mathrm{d} \Theta
\end{array}\right) .
\end{aligned}
$$

This Cartan form completes the $S^{3 \mid 4 n}$ supervielbein derived in (4.52) with its fermionic counterpart $\mathcal{E}^{\text {fer }}$ and the $\mathrm{SO}^{*}(2 n)$-connection $\omega^{\mathfrak{s 0}^{*}(2 n)}$ :

$$
\mathcal{E}^{\mathrm{fer}}=\mathbf{M}\left(\mathrm{d} \Theta-\Theta \boldsymbol{h}^{-1} \mathrm{~d} \boldsymbol{h}\right), \quad \omega^{\mathfrak{5 0} 0^{*}(2 n)}=\mathbf{M d} \mathbf{M}+\mathbf{M}^{-1} \Theta \boldsymbol{h}^{\dagger} \mathrm{d}\left(\mathbf{h} \Theta^{\dagger} \Omega \mathbf{M}^{-1}\right) .
$$

In the $n=1$ case, in which $\mathrm{SO}^{*}(2)=\mathrm{SO}(2) \cong \mathrm{U}(1)$, the $\mathrm{OSp}(2 \mid 2)$ supergroup is isomorphic to $\mathrm{SU}(2 \mid 1)$. To reduce the $\operatorname{OSp}(2 \mid 2)$ superalgebra valued element (6.24) to a corresponding $\mathrm{SU}(2 \mid 1)$ superalgebra element $\mathbf{u}$ it is convenient to use the projector $\mathbb{P}_{\Omega}=$ $\frac{1}{2}(\mathbb{1}+\Omega)$, then

$$
\mathbf{u}=\mathbb{P}_{\Omega} \mathbf{s}^{3 \mid 4} \mathbb{P}_{\Omega}=\left(\begin{array}{cc}
0 & \theta \\
-\bar{\theta} & \mathrm{ix}
\end{array}\right),
$$

where $\theta=\mathbb{P}_{\Omega} \Theta$ and $\bar{\theta}=\Omega^{\dagger} \mathbb{P}_{\Omega}=(\theta)^{\dagger}$. Note that in the realization in which $\Omega=-\sigma_{3}$ (see (5.2)), the Grassmann variables $\theta^{\alpha}$ and $\bar{\theta}_{\alpha}$ transform under the complex conjugate one-dimensional representations of $\mathrm{U}(1)$. This just reflects the fact that the rank of (6.34) is 3, i.e. the same as of the $\mathrm{SU}(2 \mid 1)$ superalgebra valued matrix generating an $\frac{\mathrm{SU}(2 \mid 1)}{\mathrm{U}(1)}$ coset element.

\section{3 $S^{3 \mid 4}$ in the matrix realization of $S U(2 \mid 1)$}

For completeness, let us now consider the description of the supersphere $S^{3 \mid 4}$ as the super$\operatorname{coset}^{17}$

$$
S^{3 \mid 4}=\frac{\mathrm{SU}(2 \mid 1)}{\mathrm{U}(1)},
$$

\footnotetext{
${ }^{17}$ For the construction of quantum mechanical models on different cosets of SU(2|1) see e.g. [34, 35] and references therein. In [34] it was shown, in particular, that $\mathrm{SU}(2 \mid 1)$ admits a supercoset which is an analog of the harmonic analytic superspace of the standard $N=4, d=1$ supersymmetry.
} 
which is the same as the supercoset $\frac{\mathrm{OSp}(2 \mid 2)}{\mathrm{SO}(2)}$. A matrix realization of the generic $\mathrm{SU}(2 \mid 1)$ supergroup element that is similar to (6.31) is

$$
U=\left(\begin{array}{cc}
\mathrm{e}^{-2 \mathrm{i} \varphi} \sqrt{1-\theta \bar{\theta}} & \mathrm{e}^{\mathrm{i} \varphi} \theta^{\beta} \\
-\frac{\mathrm{e}^{-2 \mathrm{i} \varphi} h_{\alpha} \bar{\theta}_{\gamma}}{\sqrt{1-\theta \bar{\theta}}} & \mathrm{e}^{\mathrm{i} \varphi} h_{\alpha}{ }^{\beta}
\end{array}\right),
$$

where $\alpha, \beta, \gamma=1,2$ and $\theta \bar{\theta} \equiv \theta^{\alpha} \bar{\theta}_{\alpha}=-\bar{\theta} \theta$ (i.e. the natural position of the index for $\theta$ is "up" and for $\bar{\theta}$ is "down"). The $2 \times 2$ matrix $h_{\alpha}{ }^{\beta}$ is constrained by

$$
h_{\alpha}^{\dagger \gamma} h_{\gamma}^{\beta}=\delta_{\alpha}^{\beta}-\bar{\theta}_{\alpha} \theta^{\beta} \quad \rightarrow \quad h_{\alpha}^{\dagger \beta}=\left(\delta_{\alpha}^{\gamma}-\bar{\theta}_{\alpha} \theta^{\gamma}\right) h_{\gamma}^{-1 \beta}
$$

such that

$$
\operatorname{det} h=\operatorname{det} h^{\dagger}=\frac{1}{\sqrt{1-\theta \bar{\theta}}}, \quad \operatorname{det}\left(\delta_{\alpha}^{\beta}-\bar{\theta}_{\alpha} \theta^{\beta}\right)=\frac{1}{1-\theta \bar{\theta}}=1+\theta \bar{\theta}+(\theta \bar{\theta})^{2} .
$$

One may check that

$$
\text { Ber } U=1, \quad U^{-1}=U^{\dagger} .
$$

To check (6.38) one should use the following identity $\theta^{\alpha} \theta_{\alpha} \bar{\theta}_{\beta} \bar{\theta}^{\beta}=2(\theta \bar{\theta})^{2}$ and also note that

$$
\left(\delta_{\alpha}^{\beta}-\bar{\theta}_{\alpha} \theta^{\beta}\right)^{-1}=\delta_{\alpha}^{\beta}+\bar{\theta}_{\alpha} \theta^{\beta}(1+\theta \bar{\theta}) .
$$

The Hermitian conjugate supermatrix is

$$
U^{\dagger}=\left(\begin{array}{cc}
\mathrm{e}^{2 \mathrm{i} \varphi} \sqrt{1-\theta \bar{\theta}} & -\frac{\mathrm{e}^{2 \mathrm{i} \varphi} \theta_{\gamma}^{\gamma} h_{\gamma}^{\dagger \beta}}{\sqrt{1-\theta \bar{\theta}}} \\
\mathrm{e}^{-\mathrm{i} \varphi \bar{\theta}_{\alpha}} & \mathrm{e}^{-\mathrm{i} \varphi h_{\alpha}^{\dagger \beta}}
\end{array}\right)
$$

It follows from (6.37) that one can define a unitary matrix $\hat{h}, \hat{h}^{\dagger}=\hat{h}^{-1}$, as follows

$$
\hat{h}_{\alpha}^{\beta}=h_{\alpha}^{\gamma}\left(\delta_{\gamma}^{\beta}-\bar{\theta}_{\gamma} \theta^{\beta}\right)^{-\frac{1}{2}}, \quad \sqrt{\delta_{\gamma}^{\beta}-\bar{\theta}_{\gamma} \theta^{\beta}}=\delta_{\gamma}^{\beta}-\frac{1}{2} \bar{\theta}_{\gamma} \theta^{\beta}\left(1+\frac{1}{4} \theta \bar{\theta}\right) .
$$

The supersphere $S^{3 \mid 4}$ is the coset (6.35) whose element can be identified with (6.36) at $\varphi=0$. In this realization the Hermitian Cartan form describing the geometry of $S^{3 \mid 4}$ has the following form

$$
\mathrm{i} X^{\dagger} \mathrm{d} X=\left(\begin{array}{cc}
\frac{\mathrm{i}}{2} \frac{\theta \mathcal{D} \bar{\theta}-\mathcal{D} \theta \bar{\theta}}{1-\theta \bar{\theta}} & \frac{\mathrm{i} \mathcal{D} \theta^{\beta}}{\sqrt{1-\theta \bar{\theta}}} \\
-\frac{\mathrm{i} \mathcal{D} \bar{\theta}_{\alpha}}{\sqrt{1-\theta \bar{\theta}}} & \Omega_{\alpha}{ }^{\beta}
\end{array}\right),
$$

where

$$
\omega_{\alpha}{ }^{\beta}=\mathrm{i}\left(h^{\dagger} \mathrm{d} h\right)_{\alpha}{ }^{\beta}+\mathrm{i} \bar{\theta}_{\alpha} \mathrm{d} \theta^{\beta}, \quad \mathcal{D} \theta^{\alpha}=\mathrm{d} \theta^{\alpha}+\mathrm{i} \theta^{\beta} \omega_{\beta}{ }^{\alpha}, \quad \mathcal{D} \bar{\theta}_{\alpha}=\mathrm{d} \bar{\theta}_{\alpha}-\mathrm{i} \omega_{\alpha}{ }^{\beta} \bar{\theta}_{\beta} .
$$

Note that, due to the properties (6.37) of $h_{\alpha}{ }^{\beta}$, the bosonic form $\omega_{\alpha}{ }^{\beta}$ is a Hermitian matrix $\omega^{\dagger}=\omega$. It only depends on $\mathrm{d} \theta$ which resembles a chiral basis. The $\mathrm{SU}(2 \mid 1)$ Cartan forms in a genuine chiral basis were computed in [4]. 
Splitting $\omega_{\alpha}^{\beta}$ into traceless and traceful parts, we obtain

$$
\omega_{\alpha}^{\beta}=E_{\alpha}{ }^{\beta}+\delta_{\alpha}{ }^{\beta} T,
$$

where

$$
E^{\alpha \beta}=\mathrm{i}\left(h^{\dagger} \mathrm{d} h\right)^{(\alpha \beta)}+\mathrm{i} \bar{\theta}^{(\alpha} \mathrm{d} \theta^{\beta)},
$$

is the supervielbein on $S^{3 \mid 4}$ and

$$
T=\frac{1}{2} \omega_{\alpha}^{\alpha}
$$

is the connection associated with the $\mathrm{U}(1) R$-symmetry of $S^{3 \mid 4}$, together with the upper-left term in (6.43), i.e.

$$
\tilde{T}=\frac{\mathrm{i}}{2} \frac{\theta \mathcal{D} \bar{\theta}-\mathcal{D} \theta \bar{\theta}}{1-\theta \bar{\theta}} .
$$

Using the unitary variables $\hat{h}$ defined in (6.42) one can prove that $U$ and $\tilde{U}$ are proportional to each other and have the following form

$$
\tilde{T}=2 T=\omega_{\alpha}^{\alpha}=\frac{\mathrm{i}}{2}(\theta \mathrm{d} \bar{\theta}-\mathrm{d} \theta \bar{\theta})+\mathrm{i} \theta\left(\hat{h}^{\dagger} \mathrm{d} \hat{h}\right) \bar{\theta}=\frac{\mathrm{i}}{2}(\theta \hat{\mathcal{D}} \bar{\theta}-\hat{\mathcal{D}} \theta \bar{\theta})
$$

where $\hat{\mathcal{D}} \bar{\theta}=\mathrm{d} \bar{\theta}+\left(\hat{h}^{\dagger} \mathrm{d} \hat{h}\right) \bar{\theta}$ and $\hat{\mathcal{D}} \theta=d \theta-\theta\left(\hat{h}^{\dagger} \mathrm{d} \hat{h}\right)$. Note that $\operatorname{tr}\left(\hat{h}^{\dagger} d \hat{h}\right)=0$.

In the unitary $\hat{h}$-basis for the Cartan form (6.44), the expression for $\omega_{\alpha}{ }^{\beta}$ becomes

$$
\begin{aligned}
\omega_{\alpha}{ }^{\beta}= & \mathrm{i}\left(\hat{h}^{\dagger} \mathrm{d} \hat{h}\right)_{\alpha}{ }^{\beta}-\frac{\mathrm{i}}{2}\left(\hat{\mathcal{D}} \bar{\theta}_{\alpha} \theta^{\beta}-\bar{\theta}_{\alpha} \hat{\mathcal{D}} \theta^{\beta}\right)+\frac{\mathrm{i}}{8}\left[\bar{\theta}_{\alpha} \theta^{\gamma} \mathrm{d}\left(\bar{\theta}_{\gamma} \theta^{\beta}\right)-\mathrm{d}\left(\bar{\theta}_{\alpha} \theta^{\gamma}\right) \bar{\theta}_{\gamma} \theta^{\beta}\right] \\
= & \mathrm{i}\left(\hat{h}^{\dagger} \mathrm{d} \hat{h}\right)_{\alpha}{ }^{\beta}-\frac{\mathrm{i}}{2}\left(\delta_{\alpha}^{\alpha^{\prime}}\left(1+\frac{1}{4} \theta \bar{\theta}\right)-\frac{1}{4} \bar{\theta}_{\alpha} \theta^{\alpha^{\prime}}\right)\left(\hat{\mathcal{D}} \bar{\theta}_{\alpha^{\prime}} \theta^{\beta^{\prime}}\right. \\
& \left.-\bar{\theta}_{\alpha^{\prime}} \hat{\mathcal{D}} \theta^{\beta^{\prime}}\right)\left(\delta_{\beta^{\prime}}^{\beta}\left(1+\frac{1}{4} \theta \bar{\theta}\right)-\frac{1}{4} \bar{\theta}_{\beta^{\prime}} \theta^{\beta}\right) .
\end{aligned}
$$

The off-diagonal elements of the matrix (6.43) are the fermionic vielbeins on $S^{3 \mid 4}$

$$
E^{\alpha}=\frac{\mathrm{i} \mathcal{D} \theta^{\alpha}}{\sqrt{1-\theta \bar{\theta}}}, \quad \bar{E}_{\alpha}=-\frac{\mathrm{i} \mathcal{D} \bar{\theta}_{\alpha}}{\sqrt{1-\theta \bar{\theta}}} .
$$

\section{Concluding comments and outlook}

In this paper we have described the supersphere $S^{3 \mid 4 n}$ as the three-dimensional $\mathcal{N}=2 n$ extended conformal superspace. The superconformal group $\operatorname{OSp}(2 n \mid 2,2)$ acts transitively on $S^{3 \mid 4 n}$ by fractional linear transformations, which at most scale the super-metric (4.58) being invariant under the $\operatorname{OSp}(2 n \mid 2) \times \mathrm{SU}(2)$ subgroup of $\operatorname{OSp}(2 n \mid 2,2)$. The supertwistor and bi-supertwistor realizations for $S^{3 \mid 4 n}$ developed in our paper provide all necessary prerequisites for setting up a program to compute correlations functions in off-shell superconformal field theories on $S^{3}$ in a way similar to the superspace approaches pursued in [36-39] or 
in more recent publications [40-44] which are built on the 4D bi-supertwistor construction introduced by Siegel $[22,23]$ and fully elaborated in [11]. ${ }^{18}$

A natural interesting issue for further consideration is to elaborate on peculiarities and implications of the supersymmetric and superconformal structure of Wick-rotated $\mathcal{N}$ extended supersymmetric gauge theories such as the $\mathcal{N}=4$ Gaiotto-Witten models [48] and the $\mathcal{N}=6$ ABJM model [49] put on the $S^{3}$ sphere. For instance, in Minkowski space the superconformal group of the ABJM model is $\operatorname{OSp}(6 \mid 4, \mathbb{R})$, while in the $3 D$ space of Euclidean signature its counterpart is the supergroup $\operatorname{OSp}(6 \mid 2,2)$ whose $R$-symmetry subgroup $\mathrm{SO}^{*}(6) \simeq \mathrm{SU}(3,1)$ is non-compact in contrast to the compact $R$-symmetry $\mathrm{SO}(6) \simeq \mathrm{SU}(4)$ of the theory in the Minkowski space. The two superconformal groups are different real forms of the complex supergroup $\operatorname{OSp}(6 \mid 4, \mathbb{C})$. Analogously, the $R$-symmetry group of the Euclidean $\mathcal{N}=4$ Gaiotto-Witten models should be $\mathrm{SO}^{*}(4) \simeq \mathrm{SL}(2, \mathbb{R}) \times \mathrm{SU}(2)$ for these models to be invariant under the superconformal group $\operatorname{OSp}(4 \mid 2,2)$.

It is known that the harmonic $[50,51]$ and projective [52-54] superspace approaches are most suitable for the construction of supersymmetric theories with eight supercharges in four, five and six space-time dimensions. Such superspaces are obtained by extending Minkowski superspace by auxiliary bosonic dimensions parametrizing a coset space of the compact $R$-symmetry group. In superspaces of Euclidean signature, $R$-symmetry groups are often non-compact, as is the $D=3 R$-symmetry group SO* $(2 n)$ (with $n>1$ ) considered in this paper. It is of interest to develop harmonic/projective superspace approaches to extended supersymmetric theories on $S^{3}$. The relevant mathematical formalism is sketched in appendix $\mathrm{C}$. One of the most interesting cases is $\mathcal{N}=4$. Although the corresponding $R$-symmetry group is non-compact, $\mathrm{SO}^{*}(4) \simeq \mathrm{SL}(2, \mathbb{R}) \times \mathrm{SU}(2)$, it possesses a compact coset space $S^{1} \times S^{2}$ that may be used to define nontrivial off-shell supermultiplets. This seems to be the right superspace setting in order to construct Euclidean analogs of the most general off-shell $3 D \mathcal{N}=4$ superconformal nonlinear $\sigma$-models [9].

\section{Acknowledgments}

The authors are grateful to Igor Samsonov for stimulating discussions. SMK is also grateful to Joseph Novak for reading the manuscript. The work of SMK is supported in part by the Australian Research Council projects DP1096372 and DP140103925. Work of DS was partially supported by the Padova University Project CPDA119349 and the INFN Special Initiative ST\&FI. SMK is thankful to INFN, Padova section and the Department of Physics and Astronomy "Galileo Galilei" at the University of Padova for kind hospitality at the initial stage of this project. D.S. would also like to acknowledge warm hospitality extended to him at the School of Physics of the University of Western Australian during a work-in-progress period.

\footnotetext{
${ }^{18}$ The bi-supertwistor construction of $4 \mathrm{D}$ compactified Minkowski (or conformal) superspaces was called "superembedding formalism" in [40-42]. Indeed, this construction may be viewed as a specific example of a general (super)embedding approach reviewed in [45] in application to superbranes. We also point out that there exists an alternative use of the name "conformal superspace" for the off-shell supergravity formulations developed in [46, 47].
} 


\section{A Matrix realizations of $\operatorname{Sp}(2 n, \mathbb{R})$ and $\mathrm{SO}^{*}(2 n)$}

Consider the complex symplectic group $\operatorname{Sp}(2 n, \mathbb{C})$,

$$
\operatorname{Sp}(2 n, \mathbb{C}):=\left\{g \in \mathrm{GL}(2 n, \mathbb{C}), \quad g^{\mathrm{T}} J_{n, n} g=J_{n, n}, \quad J_{n, n}=\left(\begin{array}{cc}
0 & \mathbb{1}_{n} \\
-\mathbb{1}_{n} & 0
\end{array}\right)\right\}
$$

and its subgroup $\operatorname{Sp}(2 n, \mathbb{R})$ consisting of all real symplectic matrices. ${ }^{19}$ For the latter group, there exists a different realization that is used in many applications, see, e.g., [32]. It is based on the isomorphism

$$
\mathrm{Sp}(2 n, \mathbb{R}) \cong \mathrm{Sp}(2 n, \mathbb{C}) \bigcap \mathrm{SU}(n, n)
$$

where the pseudo-unitary group $\mathrm{SU}(n, n)$ is defined by

$$
\mathrm{SU}(n, n):=\left\{g \in \mathrm{SL}(2 n, \mathbb{C}), \quad g^{\dagger} I_{n, n} g=I_{n, n}, \quad I_{n, n}=\left(\begin{array}{cc}
\mathbb{1}_{n} & 0 \\
0 & -\mathbb{1}_{n}
\end{array}\right)\right\} .
$$

To prove (A.2) one performs the similarity transformation of an $\operatorname{Sp}(2 n, \mathbb{R})$ matrix

$$
g \rightarrow h:=T g T^{-1}, \quad g \in \mathrm{Sp}(2 n, \mathbb{R}),
$$

where

$$
T=\frac{1}{\sqrt{2}}\left(\begin{array}{cc}
\mathbb{1}_{n} & \mathrm{i} \mathbb{1}_{n} \\
\mathrm{i} \mathbb{1}_{n} & \mathbb{1}_{n}
\end{array}\right)
$$

This matrix is symmetric and unitary, $T^{\dagger} T=\mathbb{1}_{2 n}$, and such that $T J_{n, n} T=J_{n, n}$ and $T J_{n, n} T^{-1}=-\mathrm{i} I_{n, n}$.

Consider now the group

$$
\mathrm{SO}^{*}(2 n)=\mathrm{SO}(2 n, \mathbb{C}) \bigcap \mathrm{Sp}(2 n, \mathbb{C}):=\left\{g \in \mathrm{Sp}(2 n, \mathbb{C}), \quad g^{\mathrm{T}} g=\mathbb{1}_{2 n}\right\},
$$

with $\operatorname{Sp}(2 n, \mathbb{C})$ defined by (A.1). This group is isomorphic to

$$
H:=\left\{h \in \mathrm{SU}(n, n), \quad h^{\mathrm{T}} I_{n, n} J_{n, n} h=I_{n, n} J_{n, n}, \quad I_{n, n} J_{n, n}=\left(\begin{array}{cc}
0 & \mathbb{1}_{n} \\
\mathbb{1}_{n} & 0
\end{array}\right)\right\} .
$$

The proof is based on considering the similarity transformation

$$
g \rightarrow h:=T g T^{-1}, \quad g \in \mathrm{SO}^{*}(2 n),
$$

with the matrix $T$ given by (A.5).

\footnotetext{
${ }^{19}$ All symplectic matrices are unimodular, $\operatorname{Sp}(2 n, \mathbb{C}) \subset \operatorname{SL}(2 n, \mathbb{C})$.
} 


\section{B Conformal spaces}

Consider a $d$-dimensional pseudo-Euclidean space $\mathbb{E}^{s, t}$ parametrized by Cartesian coordinates $x^{a}$, where $a=1, \ldots, d$, and endowed with the metric

$$
\eta_{a b}=\operatorname{diag}(1, \ldots, 1,-1, \ldots,-1),
$$

with $s>0$ 'pluses' and $t$ 'minuses' on the diagonal. The conformal algebra of $\mathbb{E}^{s, t}$ is known to be $\mathfrak{s o}(1+s, 1+t)$. It is also known that the corresponding conformal group does not act globally on $\mathbb{E}^{s, t}$. Its action is well defined on a conformal compactification $\overline{\mathbb{E}}^{s, t}$ of $\mathbb{E}^{s, t}$. Similar to the works of Veblen [16] and Dirac [17], the space $\overline{\mathbb{E}}^{s, t}$ may be introduced as follows. We consider a $(d+2)$-dimensional pseudo-Euclidean space $\mathbb{E}^{1+s, 1+1}$ with coordinates $X^{\hat{a}}=\left(X^{-1}, X^{a}, X^{d+1}\right)$ and metric

$$
\eta_{\hat{a} \hat{b}}=\left(\begin{array}{ccc}
1 & 0 & 0 \\
0 & \eta_{a b} & 0 \\
0 & 0 & -1
\end{array}\right) \text {. }
$$

Embedded into $\mathbb{E}^{1+s, 1+t}$ is the cone $\mathcal{C}$ defined by

$$
\eta_{\hat{a} \hat{b}} X^{\hat{a}} X^{\hat{b}}=0
$$

By definition, $\overline{\mathbb{E}}^{s, t}$ is the space of all straight lines belonging to $\mathcal{C}$ and passing through the origin of $\mathbb{E}^{1+s, 1+t}$. It can be defined as the quotient space of $\mathcal{C} \backslash\{0\}$ with respect to the equivalence relation

$$
X^{\hat{a}} \sim \lambda X^{\hat{a}}, \quad \lambda \in \mathbb{R} \backslash\{0\},
$$

which identifies all points on a straight line in $\mathbb{E}^{1+s, 1+t}$. The group $\mathrm{O}(1+s, 1+t)$ naturally acts on $\overline{\mathbb{E}}^{s, t}$ such that the group elements $g$ and $-g$ generate the same transformation, for any $g \in \mathrm{O}(1+s, 1+t)$. The conformal group of $\mathbb{E}^{s, t}, \operatorname{Conf}\left(\mathbb{E}^{s, t}\right)$, is defined to be $\mathrm{O}(1+s, 1+t) / \mathbb{Z}_{2}$. If $d$ is odd, the conformal group may be identified with $\mathrm{SO}(1+s, 1+t)$. The space $\overline{\mathbb{E}}^{s, t}$ is a homogeneous space of $\operatorname{Conf}\left(\mathbb{E}^{s, t}\right)$.

As a topological space, $\overline{\mathbb{E}}^{s, t}$ is homeomorphic to

$$
\begin{aligned}
\overline{\mathbb{E}}^{s, t} & =\left(S^{s} \times S^{t}\right) / \mathbb{Z}_{2}, \quad t>0 ; \\
\overline{\mathbb{E}}^{d} & \equiv \overline{\mathbb{E}}^{d, 0}=S^{d} .
\end{aligned}
$$

Indeed, for $t>0$ the constraint (B.3) and equivalence relation (B.4) can be used to choose $X^{\underline{a}}$ such that

$$
\left(X^{-1}\right)^{2}+\sum_{i=1}^{s}\left(X^{i}\right)^{2}=\sum_{i=s+1}^{d}\left(X^{i}\right)^{2}+\left(X^{d+1}\right)^{2}=1 .
$$

For such a choice, the equivalence relation (B.4) still allows us to identify $X^{\hat{a}}$ and $-X^{\hat{a}}$, which is the reason for $\mathbb{Z}_{2}$ in (B.5a). When $t=0$, we have $X^{d+1} \neq 0$ for any non-zero point on the cone $\mathcal{C}$. As a result, the equivalence relation (B.4) can be used to choose $X^{d+1}=1$, which means

$$
\left(X^{-1}\right)^{2}+\sum_{i=1}^{s}\left(X^{i}\right)^{2}=1 .
$$


Pseudo-Euclidean space $\mathbb{E}^{s, t}$ can be identified, e.g., with the open dense domain $U_{+}$ of $\overline{\mathbb{E}}^{s, t}$ on which $X^{-1}+X^{d+1} \neq 0$. This domain can be parametrized by inhomogeneous coordinates

$$
x^{a}=\frac{X^{a}}{X^{-1}+X^{d+1}},
$$

which are invariant under the identification (B.4). In terms of these coordinates, one obtains a standard action of the conformal group in $\mathbb{E}^{s, t}$. Along with $U_{+}$, we can consider the open set $U_{-}$of $\overline{\mathbb{E}}^{s, t}$ on which $X^{-1}-X^{d+1} \neq 0$. The latter may be parametrized by coordinates

$$
y^{a}=\frac{X^{a}}{X^{-1}-X^{d+1}} .
$$

In the overlap of the two charts, $U_{+} \cap U_{-}$, it holds that

$$
y^{a}=-\frac{x^{a}}{x^{2}}, \quad x^{2}=\eta_{a b} x^{a} x^{b} .
$$

In the Euclidean case, $t=0$, the charts $U_{+}$and $U_{-}$constitute an atlas of the conformal space, $S^{d}=U_{+} \cup U_{-}$.

The conformal group consists of two disjoint connected components,

$$
\operatorname{Conf}\left(\mathbb{E}^{s, t}\right)=\mathrm{SO}_{0}(1+s, 1+t) \bigcup I \cdot \mathrm{SO}_{0}(1+s, 1+t),
$$

where $I$ is a discrete transformation that may be defined as follows $I: X^{-1} \rightarrow-X^{-1}$, $X^{a} \rightarrow X^{a}, X^{d+1} \rightarrow X^{d+1}$. This conformal inversion acts on $\overline{\mathbb{E}}^{s, t}$ as

$$
x^{a} \rightarrow \frac{x^{a}}{x^{2}}
$$

\section{Fibre bundles over the supersphere}

It is possible to introduce fibre bundles over $S^{3 \mid 4 n}$ by generalizing the construction of subsection 4.2 to include odd supertwistors. ${ }^{20}$ Odd supertwistors will parametrize fibres over the supersphere. Given such an odd supertwistor $\Psi$, it is defined by the following two conditions: (i) it is orthogonal to the even supertwistors $T^{\mu}$ parametrizing $S^{3 \mid 4 n}$ with respect to the inner products (4.4),

$$
\left\langle T^{\mu} \mid \Psi\right\rangle_{\Xi}=0, \quad\left\langle T^{\mu} \mid \Psi\right\rangle_{\Upsilon}=0 ;
$$

(ii) it is defined modulo the equivalence relation

$$
\Psi \sim \Psi+T^{\mu} a_{\mu}
$$

for arbitrary $a$-numbers $a_{\mu}$ (i.e. odd elements of the Grassmann algebra). When $T^{\mu}$ are chosen as in (4.17), the equivalence relation (C.2) allows us to choose $\Psi$ to be

$$
\Psi=\left(\begin{array}{c}
v_{i} \\
\xi_{\alpha} \\
0
\end{array}\right),
$$

\footnotetext{
${ }^{20}$ Our approach in this appendix is inspired by the construction of compactified harmonic/projective superspaces with Lorentzian signature given in [9-11]. These papers built on earlier works [55-57].
} 
where $v_{i}$ is an even $2 n$-vector, and $\xi_{\alpha}$ an odd two-spinor. Imposing the orthogonality conditions (C.1) gives, respectively,

$$
\begin{aligned}
\xi & =-\left(\boldsymbol{h}^{\dagger}\right)^{-1} \Theta^{\dagger} \Omega v \\
& =\sigma_{2}\left(\boldsymbol{h}^{\mathrm{T}}\right)^{-1} \Theta^{\mathrm{T}} v .
\end{aligned}
$$

These two expressions for $\xi$ are actually equivalent due to the reality conditions (4.19). We see that $\Psi$ brings in only bosonic degrees of freedom that are described by the complex $2 n$ vector $v_{i}$. By taking several odd supertwistors and imposing $\operatorname{OSp}(2 n \mid 2,2)$ invariant conditions, the bosonic $v$-variables may be made to parametrize a homogeneous space of $\mathrm{SO}^{*}(2 n)$.

In the case of a single odd supertwistor, we may impose the following conditions

$$
\langle\Psi \mid \Psi\rangle_{\Xi}=0, \quad\langle\Psi \mid \Psi\rangle_{\Upsilon}=0 .
$$

It is easy to see that for $n=1$ the $v$-variables describe a one-sphere $S^{1}$.

Given several odd supertwistors $\Psi^{M}$, with $M=1, \ldots, m$, we may choose them to describe odd $m$ planes. Then the equivalence relation (C.2) should be replaced by a more general one of the form

$$
\Psi^{M} \sim \Psi^{N} A_{N}{ }^{M}+T^{\mu} a_{\mu}{ }^{M}, \quad A=\left(A_{M^{N}}\right) \in \mathrm{GL}(m, \mathbb{C}) .
$$

Now we may impose $\operatorname{OSp}(2 n \mid 2,2)$ invariant conditions in terms of the supermatrix $\hat{\Psi}:=$ $\left(\Psi_{A}^{M}\right)$. In particular, for $n>1$ and $m=2$ we may choose the conditions

$$
\hat{\Psi}^{\dagger} \Xi \hat{\Psi}>0, \quad \hat{\Psi}^{\mathrm{T}} \Upsilon \hat{\Psi}=0
$$

where the notation $\hat{\Psi}^{\dagger} \Xi \hat{\Psi}>0$ means that the Hermitian matrix $\hat{\Psi}^{\dagger} \Xi \hat{\Psi}$ is positive definite. For this choice the $v$-variables describe the Hermitian symmetric space $\mathrm{SO}^{*}(2 n) / \mathrm{U}(n)$, see, e.g., [32]. In the extreme case $m=2 n$, no degrees of freedom are described by the $v$-variables, since the equivalence relation (C.7) allows us to bring any odd $2 n$-plane to the form

$$
\hat{\Psi}=\left(\begin{array}{c}
\frac{\Omega}{-\left(\boldsymbol{h}^{\dagger}\right)^{-1} \Theta^{\dagger}} \\
0
\end{array}\right) .
$$

One may check that this odd $2 n$-plane is real under the star-map (4.12).

Open Access. This article is distributed under the terms of the Creative Commons Attribution License (CC-BY 4.0), which permits any use, distribution and reproduction in any medium, provided the original author(s) and source are credited.

\section{References}

[1] A. Kapustin, B. Willett and I. Yaakov, Exact results for Wilson loops in superconformal Chern-Simons theories with matter, JHEP 03 (2010) 089 [arXiv:0909.4559] [INSPIRE].

[2] D.L. Jafferis, The exact superconformal R-symmetry extremizes Z, JHEP 05 (2012) 159 [arXiv: 1012.3210] [INSPIRE]. 
[3] N. Hama, K. Hosomichi and S. Lee, Notes on SUSY gauge theories on three-sphere, JHEP 03 (2011) 127 [arXiv: 1012.3512] [INSPIRE].

[4] I.B. Samsonov and D. Sorokin, Superfield theories on $S^{3}$ and their localization, JHEP 04 (2014) 102 [arXiv: 1401.7952] [INSPIRE].

[5] S.M. Kuzenko and G. Tartaglino-Mazzucchelli, Three-dimensional $N=2$ (AdS) supergravity and associated supercurrents, JHEP 12 (2011) 052 [arXiv:1109.0496] [INSPIRE].

[6] S.M. Kuzenko, U. Lindström and G. Tartaglino-Mazzucchelli, Three-dimensional ( $p, q)$ AdS superspaces and matter couplings, JHEP 08 (2012) 024 [arXiv: 1205.4622] [INSPIRE].

[7] D. Butter, S.M. Kuzenko and G. Tartaglino-Mazzucchelli, Nonlinear $\sigma$-models with AdS supersymmetry in three dimensions, JHEP 02 (2013) 121 [arXiv:1210.5906] [INSPIRE].

[8] S.M. Kuzenko and G. Tartaglino-Mazzucchelli, $N=4$ supersymmetric Yang-Mills theories in $A d S_{3}$, JHEP 05 (2014) 018 [arXiv: 1402.3961] [INSPIRE].

[9] S.M. Kuzenko, J.-H. Park, G. Tartaglino-Mazzucchelli and R. Unge, Off-shell superconformal nonlinear $\sigma$-models in three dimensions, JHEP 01 (2011) 146 [arXiv:1011.5727] [INSPIRE].

[10] S.M. Kuzenko, On compactified harmonic/projective superspace, 5D superconformal theories and all that, Nucl. Phys. B 745 (2006) 176 [hep-th/0601177] [INSPIRE].

[11] S.M. Kuzenko, Conformally compactified Minkowski superspaces revisited, JHEP 10 (2012) 135 [arXiv:1206.3940] [InSPIRE].

[12] J. Lukierski and A. Nowicki, Superspinors and graded Lorentz groups in three-dimensions, four-dimensions and five-dimensions, Fortsch. Phys. 30 (1982) 75 [INSPIRE].

[13] J. Lukierski and A. Nowicki, Quaternionic supergroups and D $=4$ Euclidean extended supersymmetries, Annals Phys. 166 (1986) 164 [INSPIRE].

[14] K. Pilch, P. van Nieuwenhuizen and M.F. Sohnius, De Sitter superalgebras and supergravity, Commun. Math. Phys. 98 (1985) 105 [InSPIRE].

[15] J. Lukierski and A. Nowicki, All possible de Sitter superalgebras and the presence of ghosts, Phys. Lett. B 151 (1985) 382 [INSPIRE].

[16] O. Veblen, Geometry of four-component spinors, Proc. Nat. Acad. Sci. 19 (1933) 503 [INSPIRE].

[17] P.A.M. Dirac, Wave equations in conformal space, Annals Math. 37 (1936) 429 [INSPIRE].

[18] A. Ferber, Supertwistors and conformal supersymmetry, Nucl. Phys. B 132 (1978) 55 [INSPIRE].

[19] Y.I. Manin, Holomorphic supergeometry and Yang-Mills superfields, J. Sov. Math. 30 (1985) 1927.

[20] Y.I. Manin, Gauge field theory and complex geometry, Springer, Berlin Germany (1988) [INSPIRE].

[21] M. Kotrla and J. Niederle, Supertwistors and superspace, Czech. J. Phys. B 35 (1985) 602 [INSPIRE].

[22] W. Siegel, Green-Schwarz formulation of selfdual superstring, Phys. Rev. D 47 (1993) 2512 [hep-th/9210008] [INSPIRE].

[23] W. Siegel, Supermulti-instantons in conformal chiral superspace, Phys. Rev. D 52 (1995) 1042 [hep-th/9412011] [INSPIRE]. 
[24] B.S. DeWitt, Supermanifolds, Cambridge University Press, Cambridge U.K. (1992) [INSPIRE].

[25] I.L. Buchbinder and S.M. Kuzenko, Ideas and methods of supersymmetry and supergravity, or a walk through superspace, IOP, Bristol U.K. (1998) [INSPIRE].

[26] E. Cartan, The theory of spinors, Dover Publications, New York U.S.A. (1981).

[27] D.V. Volkov and V.P. Akulov, Possible universal neutrino interaction, JETP Lett. 16 (1972) 438 [Pisma Zh. Eksp. Teor. Fiz. 16 (1972) 621] [INSPIRE].

[28] D.V. Volkov and V.P. Akulov, Is the neutrino a Goldstone particle?, Phys. Lett. B 46 (1973) 109 [INSPIRE].

[29] V.P. Akulov and D.V. Volkov, Goldstone fields with spin 1/2, Theor. Math. Phys. 18 (1974) 28 [Teor. Mat. Fiz. 18 (1974) 39] [INSPIRE].

[30] A.W. Knapp, Representation theory of semisimple groups, Princeton University Press, Princeton U.S.A. (2001).

[31] M. Plyushchay, D. Sorokin and M. Tsulaia, Higher spins from tensorial charges and $\operatorname{OSp}(N \mid 2 n)$ symmetry, JHEP 04 (2003) 013 [hep-th/0301067] [INSPIRE].

[32] M. Arai, S.M. Kuzenko and U. Lindström, HyperKähler $\sigma$-models on cotangent bundles of Hermitian symmetric spaces using projective superspace, JHEP 02 (2007) 100 [hep-th/0612174] [INSPIRE].

[33] S.J. Gates, M.T. Grisaru, M. Roček and W. Siegel, Superspace or one thousand and one lessons in supersymmetry, hep-th/0108200 [INSPIRE].

[34] E. Ivanov and S. Sidorov, Deformed supersymmetric mechanics, Class. Quant. Grav. 31 (2014) 075013 [arXiv:1307.7690] [INSPIRE].

[35] E. Ivanov and S. Sidorov, Super Kähler oscillator from $\mathrm{SU}(2 \mid 1)$ superspace, J. Phys. A 47 (2014) 292002 [arXiv:1312.6821] [InSPIRE].

[36] H. Osborn, $N=1$ superconformal symmetry in four-dimensional quantum field theory, Annals Phys. 272 (1999) 243 [hep-th/9808041] [INSPIRE].

[37] J.-H. Park, Superconformal symmetry and correlation functions, Nucl. Phys. B 559 (1999) 455 [hep-th/9903230] [INSPIRE].

[38] S.M. Kuzenko and S. Theisen, Correlation functions of conserved currents in $N=2$ superconformal theory, Class. Quant. Grav. 17 (2000) 665 [hep-th/9907107] [INSPIRE].

[39] J.-H. Park, Superconformal symmetry in three-dimensions, J. Math. Phys. 41 (2000) 7129 [hep-th/9910199] [INSPIRE].

[40] W.D. Goldberger, W. Skiba and M. Son, Superembedding methods for $4 D N=1$ SCFTs, Phys. Rev. D 86 (2012) 025019 [arXiv:1112.0325] [INSPIRE].

[41] M. Maio, Superembedding methods for $4 D N$-extended SCFTs, Nucl. Phys. B 864 (2012) 141 [arXiv:1205.0389] [INSPIRE].

[42] W.D. Goldberger, Z.U. Khandker, D. Li and W. Skiba, Superembedding methods for current superfields, Phys. Rev. D 88 (2013) 125010 [arXiv:1211.3713] [INSPIRE].

[43] A.L. Fitzpatrick et al., Covariant approaches to superconformal blocks, JHEP 08 (2014) 129 [arXiv:1402.1167] [INSPIRE]. 
[44] Z.U. Khandker, D. Li, D. Poland and D. Simmons-Duffin, $N=1$ superconformal blocks for general scalar operators, JHEP 08 (2014) 049 [arXiv: 1404.5300] [INSPIRE].

[45] D.P. Sorokin, Superbranes and superembeddings, Phys. Rept. 329 (2000) 1 [hep-th/9906142] [INSPIRE].

[46] D. Butter, $N=1$ conformal superspace in four dimensions, Annals Phys. 325 (2010) 1026 [arXiv:0906.4399] [INSPIRE].

[47] D. Butter, $N=2$ conformal superspace in four dimensions, JHEP 10 (2011) 030 [arXiv:1103.5914] [INSPIRE].

[48] D. Gaiotto and E. Witten, Janus configurations, Chern-Simons couplings, and the $\theta$-angle in $N=4$ super Yang-Mills theory, JHEP 06 (2010) 097 [arXiv:0804.2907] [INSPIRE].

[49] O. Aharony, O. Bergman, D.L. Jafferis and J. Maldacena, $N=6$ superconformal Chern-Simons-matter theories, M2-branes and their gravity duals, JHEP 10 (2008) 091 [arXiv:0806.1218] [INSPIRE].

[50] A. Galperin, E. Ivanov, S. Kalitsyn, V. Ogievetsky and E. Sokatchev, Unconstrained $N=2$ matter, Yang-Mills and supergravity theories in harmonic superspace, Class. Quant. Grav. 1 (1984) 469 [Corrigendum ibid. 2 (1985) 127] [INSPIRE].

[51] A.S. Galperin, E.A. Ivanov, V.I. Ogievetsky and E.S. Sokatchev, Harmonic superspace, Cambridge University Press, Cambridge U.K. (2001).

[52] A. Karlhede, U. Lindström and M. Roček, Selfinteracting tensor multiplets in $N=2$ superspace, Phys. Lett. B 147 (1984) 297 [INSPIRE].

[53] U. Lindström and M. Roček, New HyperKähler metrics and new supermultiplets, Commun. Math. Phys. 115 (1988) 21 [InSPIRE].

[54] U. Lindström and M. Roček, $N=2$ super Yang-Mills theory in projective superspace, Commun. Math. Phys. 128 (1990) 191 [INSPIRE].

[55] A.A. Rosly, Gauge fields in superspace and twistors, Class. Quant. Grav. 2 (1985) 693 [INSPIRE].

[56] J. Lukierski and A. Nowicki, General superspaces from supertwistors, Phys. Lett. B 211 (1988) 276 [inSPIRE].

[57] P.S. Howe and G.G. Hartwell, A superspace survey, Class. Quant. Grav. 12 (1995) 1823 [INSPIRE]. 